TRANSACTIONS OF THE

AMERICAN MATHEMATICAL SOCIETY

Volume 356, Number 2, Pages 659-689

S 0002-9947(03)03239-2

Article electronically published on September 22, 2003

\title{
VARIATIONAL PRINCIPLES FOR CIRCLE PATTERNS AND KOEBE'S THEOREM
}

\author{
ALEXANDER I. BOBENKO AND BORIS A. SPRINGBORN
}

\begin{abstract}
We prove existence and uniqueness results for patterns of circles with prescribed intersection angles on constant curvature surfaces. Our method is based on two new functionals - one for the Euclidean and one for the hyperbolic case. We show how Colin de Verdière's, Brägger's and Rivin's functionals can be derived from ours.
\end{abstract}

\section{INTRODUCTION}

The subject of this paper is a special class of configurations, or patterns, of intersecting circles on constant curvature surfaces. The combinatorial aspect of such a pattern is described by a cellular decomposition of the surface. The faces of the cellular decomposition correspond to circles, and the vertices correspond to points where circles intersect. (See Figures 1 and 2.)

In the most general case that we consider, the surface may have cone-like singularities at the centers of the circles and at the points of intersection.

In particular, we treat the problem of constructing such circle patterns when the cellular decomposition is given combinatorially, the intersection angles of the circles are prescribed by a given function on the edges (and, possibly, cone angles are prescribed by functions on the faces and vertices). Using variational methods, and the "method of coherent angles", we prove existence and uniqueness results. The most fundamental of these is Theorem 3. Similar results were obtained by Bowditch [Bow91], Garrett Gar92, Rivin [Riv99], and Leibon Lei01]. Our theorem treats circle patterns on surfaces which may be flat or hyperbolic, closed surfaces or surfaces with boundary, and there may be cone-like singularities at the centers of circles and at their points of intersection. From it we deduce Rivin's theorem on ideal hyperbolic polyhedra [Riv96], and Theorem[4, which is the analogous theorem for higher genus surfaces. This higher genus analogue is new 1

Our method is based on two new functionals - one for the Euclidean case, and one for the hyperbolic case. We show how the functionals of Colin de Verdière CdV91, Brägger Brä92, and Rivin Riv94 can be derived from ours. Leibon's functional Lei01 seems to be related as well.

Received by the editors July 23, 2002.

2000 Mathematics Subject Classification. Primary 52C26; Secondary 53A30.

The research was partially supported by the Sonderforschungsbereich 288 .

${ }^{1}$ Added in proof: After the submission of this article, the authors learned of a more general theorem by Schlenker [Sch02]. 


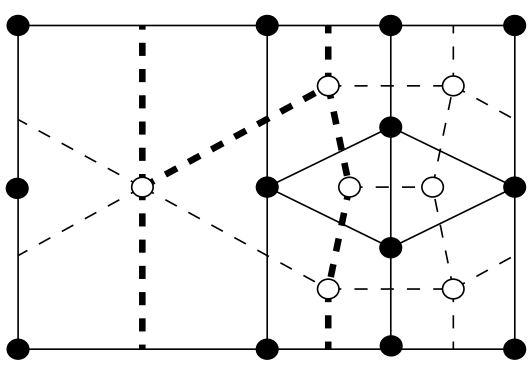

(a)

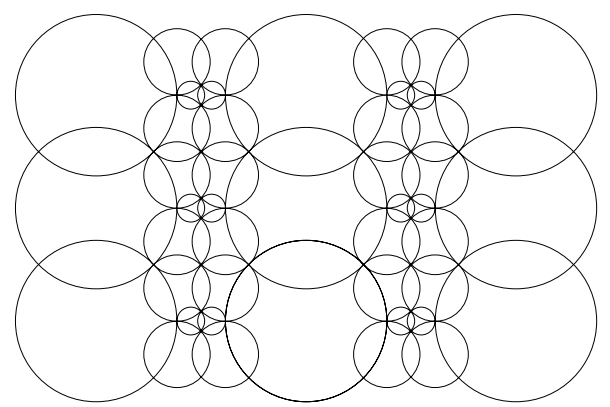

(b)

Figure 1. A cellular decomposition of the torus and the corresponding circle pattern with orthogonally intersecting circles

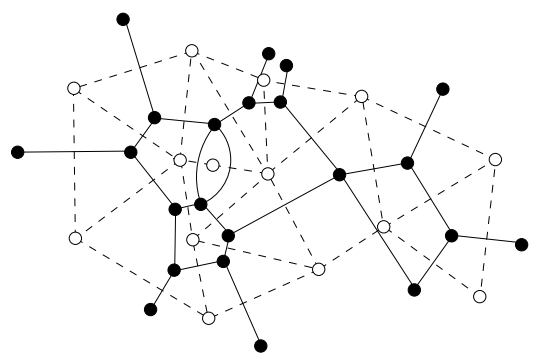

(a)

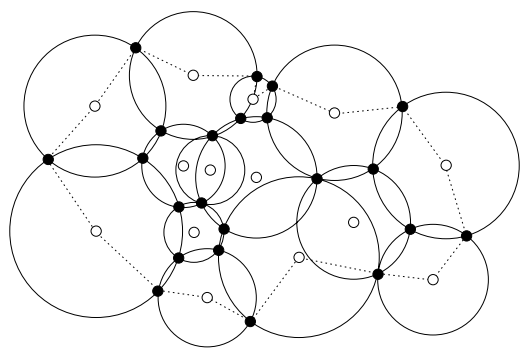

(b)

Figure 2. A cellular decomposition of the disc and a corresponding circle pattern with circles meeting at diverse angles

\section{A BRIEF SURVEY OF RELEVANT RESUlts}

A circle packing is a configuration of disjoint discs that may touch but not intersect. In 1936, Koebe published the following theorem about circle packings on the sphere [Koe36].

Theorem (Koebe). For every triangulation of the sphere there is a packing of circles on the sphere such that circles correspond to vertices and two circles touch if and only if the corresponding vertices are adjacent. This circle pattern is unique up to Möbius transformations of the sphere.

Any graph without loops or double edges, embedded in the sphere, is contained as a subgraph in the 1-skeleton of some triangulation. Hence, any such graph is the touching graph of a circle pattern on the sphere. Of course, the circle pattern is in general not unique up to Möbius transformations.

There is, however, a way to generalize Koebe's theorem to cellular decompositions of the sphere whose faces are not necessarily triangular without giving up the uniqueness part. The right class of cellular decompositions to consider is the class of cellular decompositions that are combinatorially equivalent to convex polyhedra. By a theorem of Steinitz [Ste22], SR34], these polytopal cellular decompositions are characterized by the following two conditions. First, the cellular decomposition is supposed to be regular, which means that the closure of each open cell is a closed 
disc. For the 1-cells (edges) this means that loops are not allowed. For the 2-cells this means that there are no identifications on the boundary. The second condition is that two closed cells intersect in one closed cell, if at all. This forbids double edges. More generally, it implies Steinitz' Bedingung des Nichtübergreifens: If each of two faces is incident with each of two vertices, then there is an edge that is incident with the two faces and the two vertices.

So, given a polytopal cellular decomposition of the sphere, there is a corresponding circle packing. Uniqueness is retained if an additional condition is imposed on the packing: Consider the vertices incident with a face of the cellular decomposition. The corresponding circles touch at points that correspond to the edges of the face. It is required that these points lie on a circle that intersects the original circles orthogonally. This is the content of the following theorem.

Theorem 1. For every polytopal cellular decomposition of the sphere, there exists a pattern of circles on the sphere with the following properties. There is a circle corresponding to each face and to each vertex. The vertex circles form a packing with two circles touching if and only if the corresponding vertices are adjacent. Likewise, the face circles form a packing with circles touching if and only if the corresponding faces are adjacent. For each edge, there is a pair of touching vertex circles and a pair of touching face circles. These pairs touch at the same point, intersecting each other orthogonally.

This circle pattern is unique up to Möbius transformations.

If the cellular decomposition is a triangulation, the additional condition is automatically fulfilled: three mutually touching circles have three points of contact. There is always a circle through them, and this circle intersects the three original circles orthogonally. The above theorem is therefore indeed a generalization of Koebe's theorem.

Furthermore, Theorem 1 is equivalent to the following theorem, which "probably yields the best and most powerful version of Steinitz' theorem [Zie95].'2]

Theorem 2. For every polytopal cellular decomposition of the sphere, there is a combinatorially equivalent polyhedron with edges tangent to a sphere. This polyhedron is unique up to projective transformations that fix the sphere.

There is a simultaneous realization of the dual polyhedron, such that corresponding edges of the dual and the original polyhedron touch the sphere at the same points and intersect orthogonally.

The history of Theorems 1 and 2 is somewhat unclear. Sachs' survey [Sac94] provides valuable information. However, some of the articles in preparation which are cited have never appeared in print. Thurston [Thu], who revived the interest in circle packings and circle patterns by pointing out the connection to "discrete conformal maps" and the theory of 3-manifolds, devised a method to construct some kinds of circle patterns and thus prove existence and uniqueness theorems. These methods were adapted by Brightwell and Scheinerman BS93, who state and prove Theorem 1 Schramm [Sch92] proves a theorem that is much more general than Theorem 2, In it, the sphere is replaced by an arbitrary convex body. The proof is not constructive.

\footnotetext{
${ }^{2}$ The original Steinitz theorem only asserts the existence of a three-dimensional convex polyhedron that is combinatorially equivalent to a polytopal cell decomposition of the sphere.
} 
Theorem 1 may be generalized in two directions. First, one may consider patterns of circles intersecting not orthogonally, but at arbitrary angles. There is a correspondence between such circle patterns on the sphere and polyhedra in hyperbolic space. In the Poincaré ball model, hyperbolic planes are represented by spheres that intersect the unit sphere orthogonally. Hence there is a correspondence between circles on the unit sphere and hyperbolic planes. Furthermore, the intersection angle of two circles equals the intersection angle of the corresponding planes. Orientation-preserving isometries of hyperbolic space correspond to Möbius transformations of the sphere at infinity, and vice versa. We consider circle patterns that correspond to hyperbolic polyhedra with vertices on the sphere at infinity, socalled "ideal" hyperbolic polyhedra. These are classified by the following theorem, which is due to Rivin [Riv96].

Theorem (Rivin). Let $\Sigma$ be a polytopal cellular decomposition of the sphere. Suppose an angle $\theta_{e}$, with $0<\theta_{e}<\pi$, is assigned to each edge e. There exists an ideal hyperbolic polyhedron that is combinatorially equivalent to $\Sigma$, and with exterior dihedral angles $\theta_{e}$, if and only if the following condition holds: for every cocycle $\gamma$ of edges, $\sum_{e \in \gamma} \theta_{e} \geq 2 \pi$, with equality if and only if $\gamma$ is the coboundary of a single vertex.

This ideal hyperbolic polyhedron is unique up to isometry.

Here, the exterior intersection angle of two faces of a polyhedron is the complement to $\pi$ of the interior intersection angle, which is the angle inside the polyhedron. The exterior intersection angle of two circles is the angle $\theta_{e}$ of Figure 4, it is the complement to $\pi$ of the interior intersection angle $\theta_{e}^{*}$. A cocycle is a cycle in the Poincaré dual cellular decomposition, faces of which correspond to vertices of the original decomposition, vertices to faces, and edges to edges. The concept dual to the boundary of a face is the coboundary of a vertex.

Corollary. Let $\Sigma$ be a polytopal cellular decomposition of the sphere, and suppose every vertex has $n \geq 3$ edges. Suppose that every cocycle that is not the coboundary of a single vertex is more than $n$ edges long. Then there exists, uniquely up to Möbius transformations, a circle pattern on the sphere that is combinatorially equivalent to $\Sigma$ and has exterior intersection angles $2 \pi / n$.

The case $n=4$ implies Theorem 1, and hence Theorem 2. Indeed, suppose $\Sigma$ is a polytopal cellular decomposition of the sphere. In Theorem 1, circles correspond to faces and vertices. To apply the corollary, consider the medial cellular decomposition of $\Sigma$, whose faces correspond to the faces and vertices of $\Sigma$ and whose vertices correspond to edges of $\Sigma$. Figure 3(a) shows part of a cellular decomposition of the sphere, and Figure 3(b) its medial. The dotted lines in Figure 3(a) represent the edges of the medial decomposition. In 3(b), the faces of the medial decomposition that correspond to vertices in the original decomposition are shaded. To deduce Theorem 1 from the corollary, one has to show that if we start with the polytopal cellular decomposition $\Sigma$, its medial will be polytopal, have 4-valent vertices, and all cocycles that are not coboundaries of a single vertex will be longer than 4. In fact, this is easy to see. Suffice it to remark that Steinitz' Bedingung des Nichtübergreifens for $\Sigma$ corresponds to the property of the medial that cocycles are longer than 4.

The second direction in which Theorem 1 may be generalized is to consider circle patterns not only on the sphere but also on other surfaces of constant curvature. 


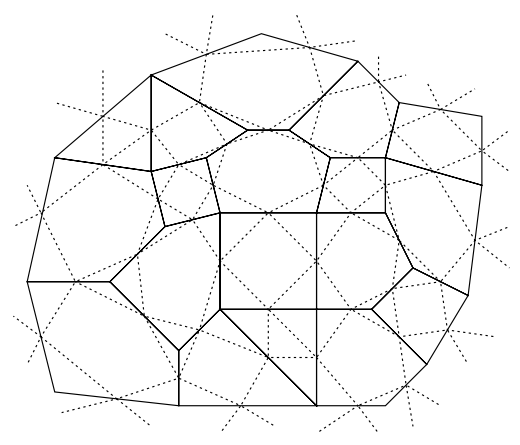

(a)

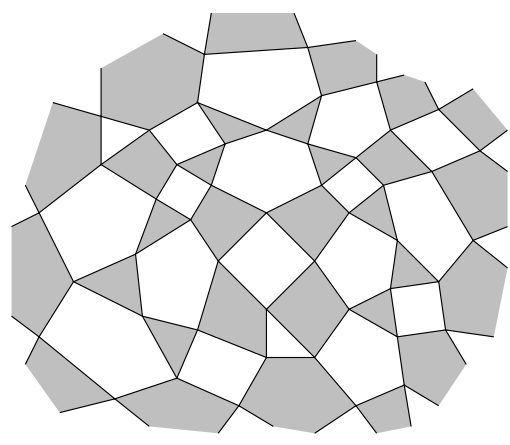

(b)

Figure 3. A cellular decomposition (a) and its medial decomposition (b)

We will consider circle patterns on closed compact surfaces of arbitrary genus, as well as circle patterns on surfaces with boundary.

It is enough to consider only orientable surfaces. To deal with non-orientable surfaces, consider first the orientable double cover; the uniqueness of the circle patterns in question will imply that the pattern on the cover can be projected down to the non-orientable surface.

By way of a further generalization, one may also consider circle patterns on surfaces which may have cone-like singularities at the centers of circles and at the intersection points.

Colin de Verdière CdV91 considers Euclidean and hyperbolic circle packings, Brägger [Brä92] only the Euclidean case. Rivin Riv94] and Leibon Lei01 treat Euclidean and hyperbolic circle patterns, respectively. They allow cone-like singularities at the vertices.

In this paper, we present new variational principles for circle patterns on Euclidean surfaces (with curvature 0) and on hyperbolic surfaces (with curvature -1). The functionals are functions of the radii of the circles. Critical points correspond to circle patterns with prescribed intersection angles and cone-like singularities. Circle patterns on the sphere are treated by projecting them stereographically to the plane and then using the Euclidean functional.

We give a complete proof of Rivin's theorem, and we prove an analogous theorem for surfaces of higher genus, Theorem 4. Both are deduced from the more fundamental, but less geometric Theorem 3 .

The idea of treating circle patterns and circle packings using a variational principle is not new. Colin de Verdière [CdV91] was the first to present a variational principle for circle packings, in which the circles correspond to the vertices of a triangulation. He treats both the Euclidean and the hyperbolic case. However, only the derivatives of the functionals are given. This suffices to prove the existence and uniqueness of circle packings. Brägger [Brä92] remarks that, investigating Colin de Verdière's functionals, one quickly arrives at the Lobachevski function. But he does not present these functionals in closed form. Instead, he derives a new variational principle. Rivin [Riv94] and Leibon [Lei01] present variational principles for circle patterns. The circles correspond to the faces of a triangulation. Rivin treats the Euclidean, Leibon the hyperbolic case. 
It was unclear how these functionals were related. We show how the functionals of Colin de Verdière, Brägger, and Rivin can be derived from our functionals. Leibon's functional seems to be related to a functional that we derive in section 6

Finally, we would like to add some remarks on the practical side of actually constructing circle patterns. The functionals of Colin de Verdière are not presented in closed form; only formulas for their derivatives are given - and in a form which seems to have prevented their integration. The functionals of Brägger, Rivin, and Leibon are presented in closed form, but their minimization has to be constrained to spaces of "coherent angle systems", which makes their application more difficult. Our functionals are given in closed form, and there are no constraints.

Thurston's proof of Koebe's theorem is also constructive. "[It is] based on a practical algorithm for actually constructing patterns of circles. The idea of the algorithm is to adjust, iteratively, the radii of the circles. A change of any single radius affects most strongly the curvature at that vertex, so this process converges reasonably well." [Thu, p. 347] Mohar shows that such an algorithm runs in polynomial time [Moh93]. Stephenson's program circlepack constructs circle packings by Thurston's method. It was used by Dubejko and Stephenson for experiments in discrete analytic function theory DS95. There are also efforts to apply such circle packing algorithms in medical imaging $\mathrm{HBS}^{+} 99$, Mar01.

Thurston's algorithm can be interpreted as a special method to minimize our functionals. Adjusting a single radius so that the neighboring circles fit around it is equivalent to minimizing our functionals in one coordinate direction.

\section{MAIN RESULtS}

After some preliminaries, we state the main results, Theorems 3 and 4 Theorem 3 is a fundamental (but somewhat technical) existence and uniqueness theorem for circle patterns. Section 4 is devoted to the proof of Theorem 3 . From it, we deduce (in section 5) Rivin's theorem and its higher genus analogue, Theorem 4

Suppose a graph is embedded in a closed topological surface such that the complement of the graph is the disjoint union of discs. This is called a 2-cell embedding of the graph. Such a 2-cell embedding of a graph gives rise to a cellular decomposition of the surface. Two decompositions are considered equivalent if one is mapped to the other by a homeomorphism of the surface. We only consider the case of oriented surfaces. A cellular surface is a cellular decomposition arising from a 2-cell embedding, considered up to homeomorphism. Associated with a cellular surface is a set $F$ of faces, a set $\vec{E}$ of oriented edges, and a set $V$ of vertices. By $E$ we denote the set of unoriented edges. For each unoriented edge in $E$, there is a pair of oppositely oriented edges in $\vec{E}$.

In the case of a surface with boundary, consider a graph embedded in the surface, such that the graph meets the boundary only at vertices, and such that the complement of the graph and the boundary is a disjoint union of open discs. This gives rise to a cellular decomposition. As the edges of the corresponding cellular surface we consider only the edges of the embedded graph. This means that there are no boundary edges, that is, every edge has a face on the left and on the right. But there are faces whose boundary is not closed, the boundary faces, and there are vertices whose coboundary is not closed, the boundary vertices.

A convenient way to represent a cellular surface $\Sigma$ jointly with its dual $\Sigma^{*}$ is the dual of the medial decomposition, $\Sigma_{q}$, which is called a quad-graph, since all its 


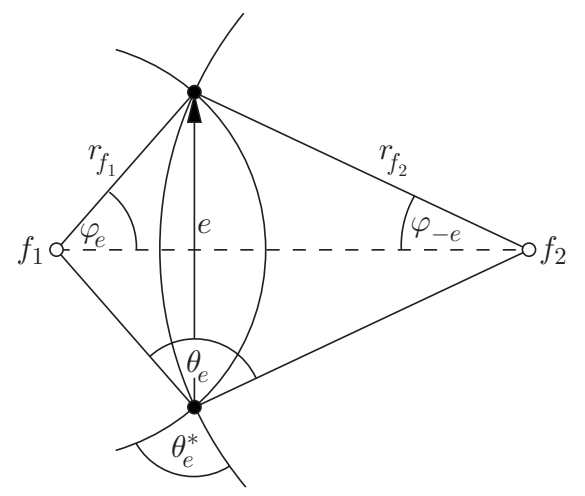

Figure 4. A Euclidean kite-shaped quadrilateral, $\theta_{e}+\theta_{e}^{*}=\pi$

faces are quadrilaterals. They correspond to the edges of $\Sigma$. There are two kinds of vertices of $\Sigma_{q}$, "white" vertices corresponding to faces of $\Sigma$ and "black" vertices corresponding to vertices of $\Sigma$. Quad-graphs turn out to be crucial for defining discrete integrable systems BS02 (in particular, those related to circle patterns) and discrete Riemann surfaces Mer01.

Suppose we are given a positive radius $r_{f}$ for each face $f$ of $\Sigma$ and an angle $\theta_{e} \in(0, \pi)$ for each non-oriented edge of $\Sigma$. For each face of $\Sigma_{q}$, construct a (Euclidean, spherical, or hyperbolic) kite-shaped quadrilateral as in Figure 4. Because corresponding sides have the same length, these kites may be glued together to form a cellular decomposition equivalent to $\Sigma_{q}$. One obtains not only a cellular decomposition of a topological manifold, but a circle pattern on a constant curvature surface with cone-like singularities. The circles correspond to faces of $\Sigma$. The vertices of $\Sigma$ correspond to points of intersection.

The cone-like singularities are at the centers of circles and at the points corresponding to vertices. The cone angle at an interior vertex $v \in V_{\Sigma}$ is

$$
\Theta_{v}=\sum \theta_{e}
$$

where the sum is taken over all oriented edges with initial vertex $v$. That is, we interpret $\theta$ as a function on the oriented edges with $\theta_{e}=\theta_{-e}$. (If an edge $e$ has $v$ as initial and terminal vertex, then $\theta_{e}$ appears twice in the sum.)

The cone angle at the center of a circle corresponding to an interior face $f$ is

$$
\Phi_{f}=2 \sum \varphi_{e}
$$

where the sum is taken over all oriented edges in the oriented boundary of $f$. An elementary geometric calculation yields, for the angles,

$$
\varphi_{e}=\frac{1}{2 i} \log \frac{r_{f_{j}}-r_{f_{k}} e^{-i \theta_{e}}}{r_{f_{j}}-r_{f_{k}} e^{i \theta_{e}}}
$$


in the Euclidean case, and

$$
\begin{aligned}
\varphi_{e}=\frac{1}{2 i}\left(\log \frac{\tanh \left(\frac{r_{f_{j}}}{2}\right)-\tanh \left(\frac{r_{f_{k}}}{2}\right) e^{-i \theta_{e}}}{\tanh \left(\frac{r_{f_{j}}}{2}\right)-\tanh \left(\frac{r_{f_{k}}}{2}\right) e^{i \theta_{e}}}\right. & \\
& \left.-\log \frac{1-\tanh \left(\frac{r_{f_{j}}}{2}\right) \tanh \left(\frac{r_{f_{k}}}{2}\right) e^{-i \theta_{e}}}{1-\tanh \left(\frac{r_{f_{j}}}{2}\right) \tanh \left(\frac{r_{f_{k}}}{2}\right) e^{i \theta_{e}}}\right)
\end{aligned}
$$

in the hyperbolic case. Here, $f_{j}$ and $f_{k}$ are the faces to the left and right of $e$.

For boundary faces and vertices, $\Phi_{f}$ and $\Theta_{v}$, given by the same formulas, are the interior angles of the boundary polygon of the quadrilateral decomposition (the dotted polygon in Figure 2(b)).

The idea is to prescribe only the intersection angles $\theta_{e}$ and the cone (or boundary) angles $\Phi_{f}$ on the faces, and to solve for the radii. The cone (or boundary) angles $\Theta_{v}$ at the vertices are determined by the intersection angles and equation (1). We will prove the following theorem.

Theorem 3. Let $\Sigma$ be an oriented cellular surface, let $\theta^{*} \in(0, \pi)^{E}$ be a function on the non-oriented edges, and let $\Phi \in(0, \infty)^{F_{\Sigma}}$ be a function on the faces.

A Euclidean circle pattern that is combinatorially equivalent to $\Sigma$, that has interior intersection angles $\theta^{*}$, and that has cone (or boundary) angles $\Phi_{f}$ at the centers of the circles exists if and only if the following two conditions are satisfied.

$$
\sum_{f \in F} \Phi(f)=\sum_{e \in E} 2 \theta^{*}(e)
$$

(ii) If $F^{\prime}$ is a nonempty subset of the face set $F, F^{\prime} \neq F$, and $E^{\prime}$ is the set of all edges incident with any face in $F^{\prime}$, then

$$
\sum_{f \in F^{\prime}} \Phi(f)<\sum_{e \in E^{\prime}} 2 \theta^{*}(e) .
$$

The Euclidean circle pattern is unique up to similarity.

A corresponding hyperbolic circle pattern on a surface of constant curvature -1 with cone-like singularities exists if and only if inequality (6) holds for all nonempty subsets $F^{\prime} \subset F$, including $F$ itself. The hyperbolic circle pattern is unique up to isometry.

This theorem is proved in section 4 .

Equation (5), which is necessary for the existence of a Euclidean circle pattern, is equivalent to the Gauss-Bonnet formula

$$
\sum_{f \in F} K_{f}+\sum_{v \in V} K_{v}=2 \pi \chi
$$

where $\chi$ is the Euler characteristic of $\Sigma$,

$$
K_{f}= \begin{cases}2 \pi-\Phi_{f} & \text { for interior faces, } \\ \pi-\Phi_{f} & \text { for boundary faces }\end{cases}
$$

and

$$
K_{v}= \begin{cases}2 \pi-\Theta_{v} & \text { for interior vertices } \\ \pi-\Theta_{v} & \text { for boundary vertices. }\end{cases}
$$


Note that the formula $\chi=|F|-|E|+|V|$ does not hold for cellular surfaces with boundary faces and boundary vertices. To get a topological invariant, one must either add boundary edges first, or count the boundary faces and vertices only half.

So far, we have not considered circle patterns in the sphere. The metric approach, focusing on the radii of circles, is less useful in this case. The reason is this: the only circle-preserving transformations of the hyperbolic plane are isometries, and those of the Euclidean plane are similarities. The Möbius transformations, which are the circle-preserving transformations of the sphere, distort the metric in a more complicated way.

However, spherical circle patterns without cone-like singularities may be treated by projecting them stereographically to the plane. This is described in detail in section 5, where, in particular, we give a proof of Rivin's theorem.

Searching for a generalization of Rivin's theorem to surfaces of higher genus, one may conjecture the condition to be that the angle sum for a coboundary is at least $2 \pi$, and equal to $2 \pi$ only for coboundaries of a single vertex. This is not the right condition, however. First, one needs to consider only the coboundaries of simply connected domains. Second, it is not enough to consider coboundaries. For example, if we cut the torus of Figure 1(a) along the dual edges represented by the heavy dashed lines, it is separated into two pieces; one of these pieces is a disc. While it is indeed necessary that the angle sum over the edges that we cut along is strictly greater than $2 \pi$, these edges do not form a boundary. To formulate the right condition, we consider cutting the dual cellular surface along some of its edges. Of the pieces into which this separates the surface, only the discs have to be considered. The angle sum over their boundary has to be at least $2 \pi$. What counts is the boundary of the pieces after cutting, not their boundary as regions in the surface.

Theorem 4. Let $\Sigma$ be a cellular surface of genus $g>0$, and let $\Sigma^{*}$ be the dual cellular surface. Suppose $\theta \in(0, \pi)^{E}$ is a function on the edges of $\Sigma$ that sums to $2 \pi$ around each vertex. We identify the edges of $\Sigma$ with the edges of $\Sigma^{*}$, and consider $\theta$ also as a function on the edges of $\Sigma^{*}$.

There exists a circle pattern on a surface of constant curvature (equal to 0 for $g=1$ and equal to -1 for $g>1$ ), combinatorially equivalent to $\Sigma$ and having exterior intersection angles $\theta$, if and only if the following condition is satisfied:

Suppose we cut $\Sigma^{*}$ along a subset of its edges, obtaining one or more pieces. For any piece that is a disc, the sum of $\theta$ over the edges in its boundary has to be at least $2 \pi$, with equality if and only if the piece consists of only one face of $\Sigma^{*}$ (vertex of $\left.\Sigma\right)$.

Both the constant curvature surface and the circle pattern are determined up to isometry if $g>1$, and up to similarity if $g=1$.

The proof is contained in section 5 .

\section{The Proof of Theorem 3}

In this section, we prove Theorem 3. Our proof is based on a variational principle for circle patterns. The corresponding functionals, one for the Euclidean and one for the hyperbolic case, are presented in section 4.1. They are functions of the radii. Of course, they also depend on the cellular surface, and the prescribed intersection angles and cone angles enter as parameters. A radius function that is a critical point 
yields a circle pattern with the prescribed intersection angles and cone angles. The functionals are strictly convex, and this implies the uniqueness claims of Theorem 3

Proving the existence claims is a little more involved. One has to estimate the functionals from below to show that they become large if some radii tend to zero or infinity. In section 4.2 we define "coherent angle systems", which are functions on the directed edges satisfying some linear equations and inequalities. We show that the functionals have minima if and only if a coherent angle system exists. This reduces the problem to the feasibility problem of linear programming, or equivalently, to the feasible flow problem of network theory.

In section 4.3, applying the feasible flow theorem of network theory, we show that the conditions of Theorem 3 are necessary and sufficient for the existence of a coherent angle system.

4.1. The functionals. Convexity and the uniqueness of circle patterns. Instead of the radii $r$, it is more convenient to use transformed variables $\rho$, the transformation being

$$
\rho= \begin{cases}\log r & \text { in the Euclidean case, } \\ \log \tanh (r / 2) & \text { in the hyperbolic case. }\end{cases}
$$

In the Euclidean case, positive radii correspond to real $\rho$. In the hyperbolic case case, positive radii correspond to negative $\rho$.

The problem is to find a function $\rho: F \rightarrow \mathbb{R}$ (which must be negative in the hyperbolic case) satisfying equation (2) with

$$
\varphi_{e}=\frac{1}{2 i} \log \frac{1-e^{\rho_{f_{k}}-\rho_{f_{j}}-i \theta_{e}}}{1-e^{\rho_{f_{k}}-\rho_{f_{j}}+i \theta_{e}}}
$$

in the Euclidean case and

$$
\varphi_{e}=\frac{1}{2 i} \log \frac{1-e^{\rho_{f_{k}}-\rho_{f_{j}}-i \theta_{e}}}{1-e^{\rho_{f_{k}}-\rho_{f_{j}}+i \theta_{e}}}-\frac{1}{2 i} \log \frac{1-e^{\rho_{f_{k}}+\rho_{f_{j}}-i \theta_{e}}}{1-e^{\rho_{f_{k}}+\rho_{f_{j}}+i \theta_{e}}}
$$

in the hyperbolic case. Here, $f_{j}$ and $f_{k}$ are the faces to the left and right of $e$, respectively. (Equations (77) and (8) are equivalent to equations (3) and (4).)

We turn this into a variational problem. The corresponding functionals are defined in terms of the imaginary part of the dilogarithm function $\mathrm{Li}_{2}$. In the appendix, all requisite facts about this function and the related Clausen integral $\mathrm{Cl}_{2}$ are summarized.

Definition. The Euclidean circle pattern functional is

$$
\begin{aligned}
S_{E u c}(\rho)=\sum_{f_{j} \circ \dot{\jmath}^{\circ} \circ f_{k}}( & \operatorname{ImLi}_{2}\left(e^{\rho_{f_{k}}-\rho_{f_{j}}+i \theta_{e}}\right)+\operatorname{ImLi}_{2}\left(e^{\rho_{f_{j}}-\rho_{f_{k}}+i \theta_{e}}\right) \\
& \left.-\theta_{e}^{*}\left(\rho_{f_{j}}+\rho_{f_{k}}\right)\right)+\sum_{\circ f} \Phi_{f} \rho_{f} .
\end{aligned}
$$

The first sum is taken over all nonoriented edges $e$, and $f_{j}$ and $f_{k}$ are the faces on either side of $e$. The summands are symmetric in $f_{j}$ and $f_{k}$; so it does not matter which face is considered as $f_{j}$ and which as $f_{k}$. The second sum is taken over all faces $f$. 
The hyperbolic circle pattern functional is, using the same notation,

$$
\begin{aligned}
S_{h y p}(\rho)=\sum_{f_{j} \circ \dot{\jmath}_{\circ} \circ f_{k}}( & \operatorname{ImLi}_{2}\left(e^{\rho_{f_{k}}-\rho_{f_{j}}+i \theta_{e}}\right)+\operatorname{ImLi}_{2}\left(e^{\rho_{f_{j}}-\rho_{f_{k}}+i \theta_{e}}\right) \\
& \left.\quad+\operatorname{ImLi}_{2}\left(e^{\rho_{f_{j}}+\rho_{f_{k}}+i \theta_{e}}\right)+\operatorname{ImLi}_{2}\left(e^{-\rho_{f_{j}}-\rho_{f_{k}}+i \theta_{e}}\right)\right) \\
& +\sum_{\circ f} \Phi_{f} \rho_{f} .
\end{aligned}
$$

Proposition 1. The critical points of the functionals $S_{E u c}$ and $S_{\text {hyp }}$ correspond to circle patterns with intersection angles $\theta$ and cone angles $\Phi$ at the centers of circles. In particular, if $\rho$ is a critical point of $S_{h y p}$, then all the $\rho_{f}$ are negative.

Proof. The functionals are of the form

$$
S(\rho)=\sum_{f_{j} \circ \bullet \circ f_{k}}\left(F_{-}\left(\rho_{f_{j}}-\rho_{f_{k}}\right)+F_{+}\left(\rho_{f_{j}}+\rho_{f_{k}}\right)\right)+\sum_{\circ f} G\left(\rho_{f}\right),
$$

where $F_{-}$is an even function. The partial derivative with respect to a face $\rho_{f_{j}}$ is

$$
\frac{\partial S}{\partial \rho_{f_{j}}}=\sum_{f_{j} \circ \mathfrak{\Upsilon}_{\bullet} \circ f_{k}}\left(F_{-}^{\prime}\left(\rho_{f_{j}}-\rho_{f_{k}}\right)+F_{+}^{\prime}\left(\rho_{f_{j}}+\rho_{f_{k}}\right)\right)+G^{\prime}\left(\rho_{f_{j}}\right),
$$

where the sum is taken over all oriented edges in the boundary of $f_{j}$. Since, by equation (38) of the appendix,

$$
\frac{d}{d x} \operatorname{Im}_{\operatorname{Li}}\left(e^{x+i \theta}\right)=\frac{1}{2 i} \log \frac{1-e^{x-i \theta}}{1-e^{x+i \theta}},
$$

one finds that

$$
\frac{\partial S}{\partial \rho_{f}}=-2\left(\sum \varphi_{e}\right)+\Phi_{f}
$$

where the sum is taken over all directed edges in the boundary of $f$.

It remains to show that if $\rho$ is a critical point of $S_{h y p}$, then all $\rho_{f}$ are negative. The function

$$
f(x)=\frac{1}{2 i} \log \frac{1-e^{x-i \theta_{e}}}{1-e^{x+i \theta_{e}}}
$$

is strictly increasing for $0<\theta<\pi$. Hence, if, in equation (8), $\rho_{f_{j}}$ is nonnegative, then $\varphi_{e}$ is nonpositive. Therefore, if some $\rho_{f}$ is nonnegative, then, from equation (12),

$$
\frac{\partial S_{h y p}}{\partial \rho_{f}} \geq \Phi(f)>0, \quad \text { if } \quad \rho_{f} \geq 0
$$

Rescaling all radii of a Euclidean circle pattern by a factor $h$ corresponds to the transformation $\rho \mapsto \rho+h 1_{F}$, where $1_{F}$ is the function that is constantly 1 on $F$. Note that

$$
S_{E u c}\left(\rho+h 1_{F}\right)=S_{E u c}(\rho)+h\left(\sum_{f \in F} \Phi_{f}-2 \sum_{e \in E} \theta_{e}^{*}\right) .
$$


The functional can have a critical point only if the coefficient of $h$ vanishes. In this case, the functional is scale-invariant, and one may restrict minimization to the subspace

$$
U=\left\{\rho \in \mathbb{R}^{F} \mid \sum_{f \in F} \rho_{f}=0\right\} .
$$

Proposition 2. The hyperbolic functional is strictly convex. The Euclidean functional is strictly convex on the subspace $U$. This implies, in the hyperbolic case, the uniqueness of circle patterns with prescribed intersection angles and cone angles, and, in the Euclidean case, uniqueness up to scale.

Proof. Taking second derivatives on both sides of equation (11), one obtains

$$
\begin{aligned}
S^{\prime \prime}= & \sum_{f_{j} \circ \bullet_{\circ} f_{k}}\left(F_{-}^{\prime \prime}\left(\rho_{f_{j}}-\rho_{f_{k}}\right)\left(d \rho_{f_{j}}-d \rho_{f_{k}}\right)^{2}+F_{+}^{\prime \prime}\left(\rho_{f_{j}}+\rho_{f_{k}}\right)\left(d \rho_{f_{j}}+d \rho_{f_{k}}\right)^{2}\right) \\
& +\sum_{\circ f} G^{\prime \prime}\left(\rho_{f}\right) d \rho_{f}^{2} .
\end{aligned}
$$

Thus, by a straightforward calculation,

$$
S_{E u c}^{\prime \prime}=\sum_{f_{j} \circ \mathfrak{\bullet}_{\circ f_{k}}} \frac{\sin \theta_{e}}{\cosh \left(\rho_{f_{k}}-\rho_{f_{j}}\right)-\cos \theta_{e}}\left(d \rho_{f_{k}}-d \rho_{f_{j}}\right)^{2}
$$

and

$$
\begin{aligned}
S_{h y p}^{\prime \prime}=\sum_{f_{j} \circ \dot{0}_{\circ} \circ f_{k}}\left(\frac{\sin \theta_{e}}{\cosh \left(\rho_{f_{k}}-\rho_{f_{j}}\right)-\cos \theta_{e}}\left(d \rho_{f_{j}}-d \rho_{f_{k}}\right)^{2}\right. \\
\\
\left.\quad+\frac{\sin \theta_{e}}{\cosh \left(\rho_{f_{j}}+\rho_{f_{k}}\right)-\cos \theta_{e}}\left(d \rho_{f_{j}}+d \rho_{f_{k}}\right)^{2}\right) .
\end{aligned}
$$

These second derivatives are positive definite on $U$ and $\mathbb{R}^{F}$, respectively.

Remark. Formulas (9) and (10) for $S_{E u c}$ and $S_{\text {hyp }}$ involve the imaginary part of the dilogarithm of a complex argument. The formulas below involve only real functions. Since, moreover, Clausen's integral is periodic, these formulas are well suited for the practical evaluation of the functionals.

For $\rho \in \mathbb{R}^{F}$, let $p$ and $s$ be the functions on oriented edges defined by

$$
p_{e}=2 \arctan \left(\tan \left(\frac{\theta_{e}^{*}}{2}\right) \tanh \left(\frac{\rho_{f_{k}}-\rho_{f_{j}}}{2}\right)\right)
$$

and

$$
s_{e}=2 \arctan \left(\tan \left(\frac{\theta_{e}^{*}}{2}\right) \tanh \left(\frac{\rho_{f_{k}}+\rho_{f_{j}}}{2}\right)\right),
$$

where $f_{j}$ and $f_{k}$ are the faces to the left and right of $e$. Note that $s_{e}=s_{-e}$ (so that $s$ may be considered as a function on non-oriented edges), but $p_{e}=-p_{-e}$. 
The following formulas hold:

$$
\begin{aligned}
S_{E u c}(\rho)=\sum_{f_{j} \circ j_{\circ} \circ f_{k}}\left(p_{e}\left(\rho_{f_{k}}-\rho_{f_{j}}\right)+\mathrm{Cl}_{2}\left(\theta_{e}^{*}+p_{e}\right)\right. & +\mathrm{Cl}_{2}\left(\theta_{e}^{*}-p_{e}\right)-\mathrm{Cl}_{2}\left(2 \theta_{e}^{*}\right) \\
& \left.-\theta_{e}^{*}\left(\rho_{f_{j}}+\rho_{f_{k}}\right)\right)+\sum_{\circ f} \Phi_{f} \rho_{f}
\end{aligned}
$$

and

$$
\begin{aligned}
S_{h y p}(\rho)=\sum_{f_{j} \circ \dot{j}_{0 f_{k}}}\left(p_{e}\left(\rho_{f_{k}}-\rho_{f_{j}}\right)+\mathrm{Cl}_{2}\left(\theta_{e}^{*}+p_{e}\right)\right. & +\mathrm{Cl}_{2}\left(\theta_{e}^{*}-p_{e}\right) \\
+s_{e}\left(\rho_{f_{j}}+\rho_{f_{k}}\right)+\mathrm{Cl}_{2}\left(\theta_{e}^{*}+s_{e}\right)+ & \mathrm{Cl}_{2}\left(\theta_{e}^{*}-s_{e}\right) \\
& \left.-2 \mathrm{Cl}_{2}\left(2 \theta_{e}^{*}\right)\right)+\sum_{\circ f} \Phi_{f} \rho_{f} .
\end{aligned}
$$

The first sum in each equation is taken over non-oriented edges, or rather, over one oriented representative $e$ for each non-oriented edge. Since both $p_{e}$ and $\left(f_{k}-f_{j}\right)$ change sign when $-e$ is chosen instead of $e$, the summands are independent of which choice is made.

These formulas can be derived using equation (40) of the appendix and some simple properties of Clausen's integral, which are also found there. 6

The hyperbolic functional will reappear in this form in equation (35) of section

4.2. Coherent angle systems and the existence of circle patterns. In section 4.1, the uniqueness of a circle pattern was deduced from the convexity of the functionals. The rest of section 4 is devoted to the existence part of Theorem 3 To establish that the functionals have a minimum, we show that they tend to infinity as $\rho$ tends to infinity in $\mathbb{R}$ or $U$, respectively.

The main difficulty in estimating the functionals from below is to somehow merge the sum over edges with the sum over faces in equations (9) and (10). This is achieved with the help of a so-called "coherent angle system". Therefore, in this section we prove that the functionals have minima if and only if coherent angle systems exist. In section 4.3 we show that the conditions of Theorem 3 are necessary and sufficient for the existence of a coherent angle system.

Definition. A coherent angle system is a function $\varphi \in \mathbb{R}^{\vec{E}}$ on the oriented edges satisfying, in the Euclidean case,

(1) for all oriented edges $e \in \vec{E}$,

$$
\varphi_{e}>0 \text { and } \varphi_{e}+\varphi_{-e}=\theta_{e}^{*}
$$

(2) for all oriented faces $f \in F$,

$$
\sum 2 \varphi_{e}=\Phi_{f}
$$

where the sum is taken over all oriented edges in the oriented boundary of $f$. In the hyperbolic case, condition (11) has to be changed to

(11) for all oriented edges $e \in \vec{E}$,

$$
\varphi_{e}>0 \text { and } \varphi_{e}+\varphi_{-e}<\theta_{e}^{*} .
$$


Proposition 3. The functional $S_{E u c}\left(S_{h y p}\right)$ has a critical point if and only if a Euclidean (hyperbolic) coherent angle system exists.

Proof. If the functional $S_{E u c}\left(S_{h y p}\right)$ has a critical point $\rho$, then equation (17) (equation (8) ) defines a coherent angle system. It remains to show that, conversely, the existence of a coherent angle system implies the existence of a critical point.

Consider first the Euclidean case. Suppose a Euclidean coherent angle system $\varphi$ exists. One easily deduces equation (5). Hence, the functional $S_{E u c}$ is scaleinvariant. We will show that $S_{E u c}(\rho) \rightarrow \infty$ if $\rho \rightarrow \infty$ in the subspace $U$ defined in equation (14). More precisely, we will show that for $\rho \in U$,

$$
S_{E u c}(\rho)>2 \min _{e \in \vec{E}} \varphi_{e} \max _{f \in F}\left|\rho_{f}\right| \text {. }
$$

The functional $S_{E u c}$ must therefore attain a minimum, which is a critical point.

For $x \in \mathbb{R}$ and $0<\theta<\pi$,

$$
\operatorname{ImLi}_{2}\left(e^{x+i \theta}\right)+\operatorname{ImLi}_{2}\left(e^{-x+i \theta}\right)>(\pi-\theta)|x|,
$$

and hence

$$
S_{E u c}(\rho)>-2 \sum_{e \in E} \theta_{e}^{*} \min \left(\rho_{f_{k}}, \rho_{f_{j}}\right)+\sum_{f \in F} \Phi_{f} \rho_{f} .
$$

Throughout this section, " $\sum_{e \in E}$ " is meant to indicate that the sum is taken over one representative from each pair of oppositely oriented edges; it does not matter which. The faces on either side of $e$ are $f_{j}$ and $f_{k}$.

Now we use the coherent angle system to merge the two sums. Because

$$
\sum_{f \in F} \Phi_{f} \rho_{f}=2 \sum_{e \in E}\left(\varphi_{e} \rho_{f_{j}}+\varphi_{-e} \rho_{f_{k}}\right)
$$

one obtains

$$
S_{E u c}(\rho)>2 \sum_{e \in E} \min \left(\varphi_{e}, \varphi_{-e}\right)\left|\rho_{f_{k}}-\rho_{f_{j}}\right| .
$$

Since we assume the cellular surface to be connected, we get

$$
S_{E u c}(\rho)>2 \min _{e \in \vec{E}} \varphi_{e}\left(\max _{f \in F} \rho_{f}-\min _{f \in F} \rho_{f}\right),
$$

and from this the estimate (17).

The hyperbolic case is similar. One shows that, if all $\rho_{f}<0$, then

$$
S_{h y p}(\rho)>2 \min _{e \in E}\left|\varphi_{e}+\varphi_{-e}-\theta_{e}^{*}\right| \max _{f \in F}\left|\rho_{f}\right| .
$$

Since it was shown in section 4.1 that $\partial S_{h y p} / \partial \rho_{f}>0$ if $\rho_{f} \geq 0$, this implies the existence of a minimum.

4.3. Conditions for the existence of coherent angle systems. With this section we complete the proof of Theorem 3 All that is left to show is the following proposition.

Proposition 4. A coherent angle system exists if and only if the conditions of Theorem 3 hold.

Proof. It is easy to see that these conditions are necessary. To prove that they are sufficient, we apply the feasible flow theorem of network theory. Let $(N, X)$ be a network (i.e., a directed graph), where $N$ is the set of nodes and $X$ is the set of branches. For any subset $N^{\prime} \subset N$, let $e x\left(N^{\prime}\right)$ be the set of branches having their 


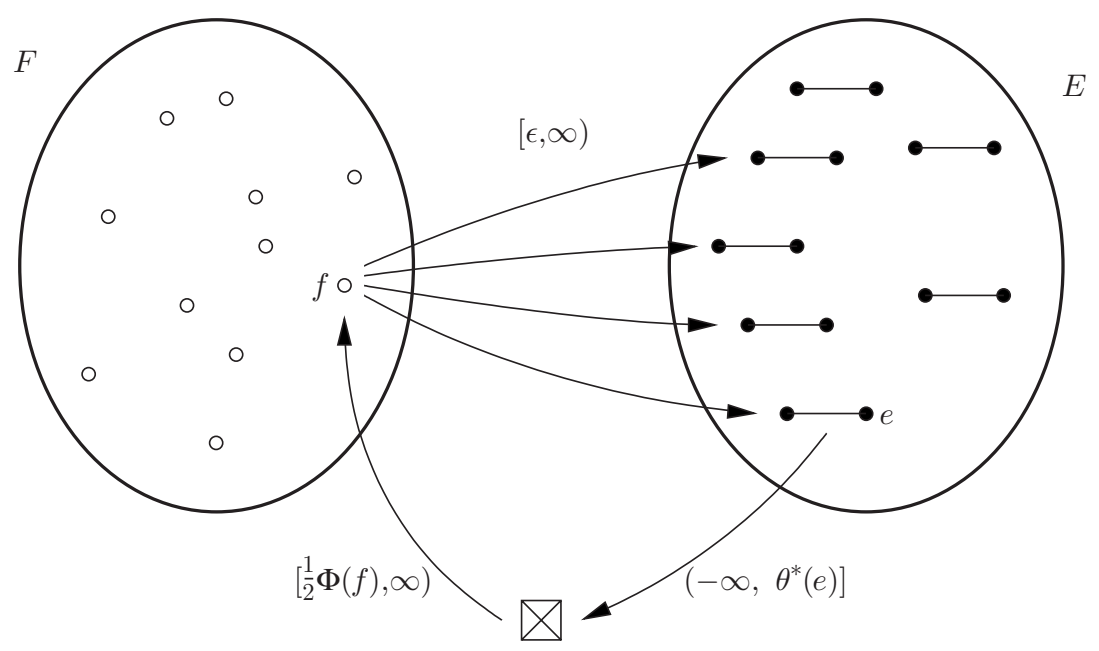

Figure 5. The network $(N, X)$. Only a few of the branches and capacity intervals are shown.

initial node in $N^{\prime}$ but not their terminal node. Let in $\left(N^{\prime}\right)$ be the set of branches having their terminal node in $N^{\prime}$ but not their initial node. Assume that there are a lower capacity bound $a_{x}$ and an upper capacity bound $b_{x}$ associated with each branch $x$, with $-\infty \leq a_{x} \leq b_{x} \leq \infty$.

Definition. A feasible flow is a function $\varphi \in \mathbb{R}^{X}$ such that Kirchhoff's current law is satisfied, i.e., for each $n \in N$,

$$
\sum_{x \in e x(\{n\})} \varphi_{x}=\sum_{x \in \operatorname{in}(\{n\})} \varphi_{x}
$$

and $a_{x} \leq \varphi_{x} \leq b_{x}$ for all branches $x$.

Feasible Flow Theorem. A feasible flow exists if and only if, for every nonempty subset $N^{\prime} \subset N$ of nodes with $N^{\prime} \neq N$,

$$
\sum_{x \in e x\left(N^{\prime}\right)} b_{x} \geq \sum_{x \in \operatorname{in}\left(N^{\prime}\right)} a_{x}
$$

A proof is given by Ford and Fulkerson [FF62, Ch. II, §3]. (Ford and Fulkerson assume the capacity bounds to be nonnegative, but this is not essential.)

To prove the proposition in the Euclidean case, consider the following network (see Figure 51). The nodes are all faces and non-oriented edges of the cellular surface, and one further node that we denote by $\nabla: N=F \cup E \cup\{\otimes\}$. There is a branch in $X$ going from $\otimes$ to each face $f \in F$ with capacity interval $\left[\frac{1}{2} \Phi_{f}, \infty\right)$. From each face $f$ there is a branch in $X$ going to the non-oriented edges of the boundary of $f$ with capacity interval $[\epsilon, \infty)$, where $\epsilon>0$ will be determined later. Finally, there is a branch in $X$ going from each non-oriented edge $e \in E$ to $\bigotimes$ with capacity $\left(-\infty, \theta_{e}^{*}\right]$.

A feasible flow in the network yields a coherent angle system. Indeed, Kirchhoff's current law at $\square$ implies that the flow into each face $f$ is $\frac{1}{2} \Phi_{f}$ and the flow out of each edge $e$ is $\theta_{e}^{*}$. It follows that the flow in the branches from $F$ to $E$ constitutes a coherent angle system. 
Assume that the conditions of Theorem [3 are fulfilled. We need to show that the condition of the feasible flow theorem is satisfied. Suppose $N^{\prime}$ is a nonempty proper subset of $N$. Let $F^{\prime}=N^{\prime} \cap F$ and $E^{\prime}=N^{\prime} \cap E$.

Consider first the case that $\otimes \in N^{\prime}$, which is the easy one. Since $N^{\prime}$ is a proper subset of $N$, there is a face $f \in F$ or an edge $e \in E$ that is not in $N^{\prime}$. In the first case there is a branch out of $N^{\prime}$ with infinite upper capacity bound. In the second case there is a branch into $N^{\prime}$ with negative infinite lower capacity bound. Either way, the condition of the feasible flow theorem is trivially fulfilled.

Now consider the case that $\otimes \notin N^{\prime}$. We may assume that for each face $f \in F^{\prime}$, the edges of the boundary of $E$ are in $E^{\prime}$. Otherwise there are branches out of $N^{\prime}$ with infinite upper capacity bound. For subsets $A, B \subset N$, denote by $A \rightarrow B$ the set of branches in $X$ having initial node in $A$ and terminal node in $B$. Then the condition of the feasible flow theorem is

$$
\sum_{f \in F^{\prime}} \frac{1}{2} \Phi_{f}+\epsilon\left|F \backslash F^{\prime} \rightarrow E^{\prime}\right| \leq \sum_{e \in E^{\prime}} \theta_{e}^{*} .
$$

It is fulfilled if we choose

$$
\epsilon<\frac{1}{2|E|} \min _{F^{\prime}}\left(\sum_{e \in E^{\prime}\left(F^{\prime}\right)} \theta_{e}^{*}-\sum_{f \in F^{\prime}} \frac{1}{2} \Phi_{f}\right),
$$

where the minimum is taken over all proper nonempty subsets $F^{\prime}$ of $F$, and where $E^{\prime}\left(F^{\prime}\right)$ is the set of all non-oriented edges incident with a face in $F^{\prime}$. The minimum is greater than zero because of condition (ii) of Theorem 3

In the hyperbolic case, the proof is only a little bit more complicated. In the network, the flow in the branches going from $\otimes$ to a face $f$ must be constrained to be exactly $\frac{1}{2} \Phi(f)$, and the capacity interval of branches going from an edge $e$ to $\square$ has to be changed to $\left(-\infty, \theta^{*}(e)-\epsilon\right]$.

\section{Circle patterns in the sphere, Rivin's theorem, AND ITS HIGHER GENUS ANALOGUE}

In section 5.1 we adapt Theorem 3 to circle patterns on the sphere. Then we use it to prove Rivin's theorem in section 5.2. Section 5.3 is devoted to the proof of Theorem 4, the higher genus analogue.

5.1. Circle patterns in the sphere. A circle pattern in the sphere may be projected stereographically to the plane, choosing some vertex $v_{\infty}$ as the center of projection. For example, Figure 6(a) shows the circle pattern combinatorially equivalent to the cube and with intersection angles $\pi / 3$ after stereographic projection.

One obtains a circle pattern in the plane in which some circles (those corresponding to faces incident with $v_{\infty}$ ) have degenerated to straight lines. Since stereographic projection is conformal, the intersection angles are the same. Furthermore, a Möbius-equivalent circle pattern in the sphere leads to a planar pattern which is similar, provided the same vertex is chosen as the center of projection.

The idea is to construct a circle pattern in the sphere by constructing the corresponding planar pattern using the Euclidean functional and then projecting it to the sphere.

To construct the planar pattern, we first remove from the cellular surface the faces incident with $v_{\infty}$ and all edges incident with them. Some of the remaining 


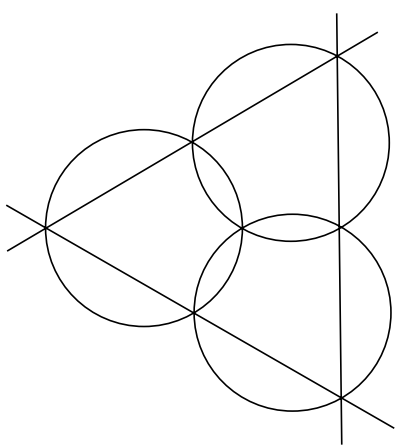

(a)

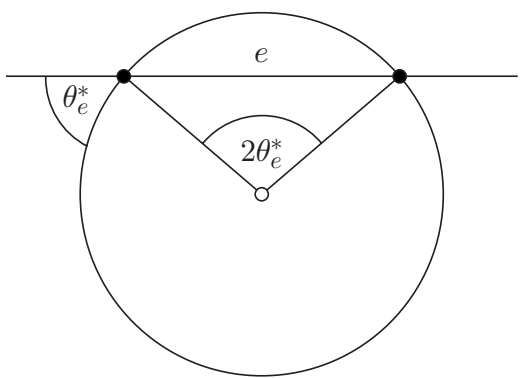

(b)

FiguRE 6. (a) The regular cubical pattern after stereographic projection. (b) How to calculate the $\Phi_{f}$ for the new boundary circles.

faces will then be boundary faces. For such a new boundary face $f$ we set

$$
\Phi_{f}=2 \pi-\sum 2 \theta_{e}^{*}
$$

where the sum is taken over all removed edges incident with $f$; see Figure6(b). For all interior faces $f$, we set

$$
\Phi_{f}=2 \pi .
$$

Assuming that the conditions of Theorem 3 are satisfied, we then construct the corresponding planar pattern, add the lines corresponding to the removed faces, and project to the sphere.

In this way, we deduce the following proposition from Theorem [3]

Proposition 5. Let $\Sigma$ be a closed cellular surface of genus 0 , and let an angle $\theta \in(0, \pi)^{E}$ be assigned to each non-oriented edge. Suppose that for each vertex $v \in V$, the sum of $\theta$ over the edges around $v$ is $2 \pi$, so that there is no curvature in the vertices. Let $\theta^{*}=\pi-\theta$.

Suppose $v_{\infty} \in V$, and let $F_{\infty}$ be the set of all faces incident with $v_{\infty}$. Suppose the following conditions hold.

(i) The 1-skeleton of the Poincaré dual $\Sigma^{*}$ remains connected if the faces in $F_{\infty}$ and all edges incident with them are removed.

$$
2 \pi\left(|F|-\left|F_{\infty}\right|\right)=\sum 2 \theta^{*}(e),
$$

where the sum is taken over all edges incident with a face in $F \backslash F_{\infty}$, i.e., all edges not incident with $v_{\infty}$.

(iii) If $F^{\prime}$ is a nonempty subset of $F \backslash F_{\infty}$ and $F^{\prime} \neq F \backslash F_{\infty}$, and $E^{\prime}$ is the set of all edges incident with a face in $F^{\prime}$, then

$$
2 \pi\left|F^{\prime}\right|<\sum_{e \in E^{\prime}} 2 \theta^{*}(e) .
$$

Then there exists a circle pattern in the sphere that is combinatorially equivalent to $\Sigma$ and that has intersection angles $\theta^{*}$. This circle pattern is unique up to Möbius transformations. 
Proof. By condition (i), after we remove from $\Sigma$ all faces in $F_{\infty}$ and all edges incident with them, we are left either with a single face, or with a connected cellular surface $\Sigma_{0}$ with boundary faces and boundary vertices. The first case is elementary. In the second case, we want to apply Theorem 3 .

For boundary and interior faces $f$ of $\Sigma_{0}$, define $\Phi_{f}$ by equations (19) and (20), respectively. We have to show that $\Phi_{f}>0$ for boundary faces. Suppose $f$ is a boundary face of $\Sigma_{0}$. Let $F^{\prime}=F \backslash\left(F_{\infty} \cup\{f\}\right)$. Subtracting (21) and (22), one obtains $2 \pi>\sum 2 \theta^{*}$, where the sum is taken over all removed edges incident with $f$. Hence $\Phi_{f}>0$.

We apply Theorem 3 to the cellular surface $\Sigma_{0}$ with intersection angles given by $\theta$ restricted to the edges of $\Sigma_{0}$ and with $\Phi$ as defined above. Conditions (ii) and (iii) of the proposition imply conditions (i) and (ii) of Theorem 3. Indeed, the face set of $\Sigma_{0}$ is $F \backslash F_{\infty}$, and

$$
\sum_{f \in F \backslash F_{\infty}} \Phi_{f}=2 \pi\left(|F|-\left|F_{\infty}\right|\right)-\sum 2 \theta_{e}^{*}
$$

where the sum on the right-hand side is taken over all edges of $\Sigma$ that are not edges of $\Sigma_{0}$ but are incident with a face in $F \backslash F_{\infty}$. Hence, condition (ii) of the proposition implies

$$
\sum_{f \in F \backslash F_{\infty}} \Phi_{f}=\sum 2 \theta_{e}^{*}
$$

where the sum is taken over all edges of $\Sigma_{0}$, i.e., condition (i) of Theorem 3

Similarly, condition (iii) of the proposition implies condition (ii) of Theorem 3 Therefore, the corresponding planar circle pattern exists, and by the construction described above, we can construct the spherical circle pattern.

5.2. A proof of Rivin's theorem. We now give a proof of Rivin's theorem. First, we show that the condition of Rivin's theorem is necessary. If $\gamma$ is the coboundary of a single vertex, then $\sum_{e \in \gamma} \theta_{e}=2 \pi$ simply means that the corresponding circle pattern has no curvature at that vertex. Suppose a circle pattern on the sphere is given and $\gamma$ is a closed copath that does not bound a single vertex. Without loss of generality, we may assume that $\gamma$ is simple, i.e., that it meets a face at most once.

Suppose that $\gamma$ does not meet all the faces of the cellular decomposition; we will deal with the other case afterwards. Then there is a point in the sphere that lies outside of all the circles belonging to $\gamma$. (In fact, each circle of a circle pattern contains points that are not contained in any of the other circles.) Project the circle pattern stereographically to the plane, using such a point as the center of projection. One obtains a sequence of intersecting circles as in Figure 7. Now

$$
\sum\left(\pi-\alpha_{j}-\beta_{j}-\gamma_{j}\right)=2 \pi
$$

and

$$
\theta_{j}=\pi-\alpha_{j}-\beta_{j} .
$$

This implies $\sum \theta_{j} \geq 2 \pi$, with equality if and only if all $\gamma_{j}$ are zero. This is the case only if the copath bounds a single vertex. 


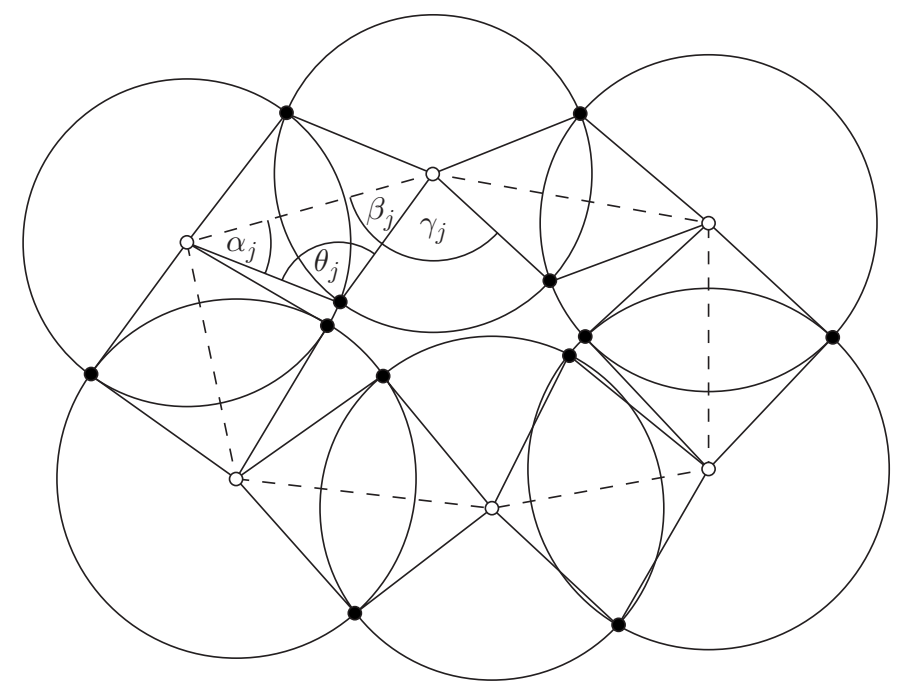

FiguRE 7. The circles and quadrilaterals corresponding to a simple closed copath after stereographic projection

Now suppose that the copath $\gamma$ does meet all the faces of the cellular decomposition. Consider the graph $(V, E \backslash \gamma)$, consisting of all vertices of the cellular decomposition and all edges that do not appear in $\gamma$. This graph is the disjoint union of two trees. Therefore, $|V|-|E \backslash \gamma|=2$. Since $\theta$ sums to $2 \pi$ around each vertex,

$$
2 \pi|V|=\sum_{e \in E} 2 \theta_{e}
$$

With $\theta_{e}<\pi$ one obtains

$$
\sum_{\gamma} 2 \theta_{e}=2 \pi|V|-\sum_{E \backslash \gamma} 2 \theta_{e}>2 \pi(|V|-|E \backslash \gamma|),
$$

and hence the desired inequality. Hence the condition of Rivin's theorem is necessary for the existence of a circle pattern.

Now, using Proposition 5, we show that the condition of Rivin's theorem is sufficient for the existence of a circle pattern. Thus we assume that $\sum_{e \in \gamma} \theta_{e} \geq 2 \pi$ for every copath $\gamma$, with equality if and only if $\gamma$ is the coboundary of a single vertex. We need to deduce the conditions of Proposition [5. Condition (i) follows from the assumption that $\Sigma$ is polytopal.

To deduce conditions (ii) and (iii), suppose that $F^{\prime}$ is a subset of $F \backslash F_{\infty}$, and that $E^{\prime}$ is the set of all edges of $\Sigma$ incident with a face in $F^{\prime}$. Consider $F^{\prime \prime}=F \backslash F^{\prime}$, the complement of $F^{\prime}$, and $E^{\prime \prime}=E \backslash E^{\prime}$, the complement of $E^{\prime}$. For each edge in $E^{\prime \prime}$, the faces on either side are in $F^{\prime \prime}$. Now consider the dual cellular decomposition $\Sigma^{*}$, and in it the graph $\Gamma=\left(F^{\prime \prime}, E^{\prime \prime}\right)$ with vertex set $F^{\prime \prime}$ and edge set $E^{\prime \prime}$. As for any graph, we have

$$
\left|E^{\prime \prime}\right|-\left|F^{\prime \prime}\right|=c-n,
$$

where $n$ is the number of connected components of $\Gamma$ and $c$ is the dimension of the cycle space. Since the graph is embedded in $\Sigma^{*}$, a cellular decomposition of the 
sphere, we have

$$
c=r-1,
$$

where $r$ is the number of regions into which $\Gamma$ separates $\Sigma^{*}$. Since $E^{\prime \prime}$ contains the edges of $\Sigma$ incident with $v_{\infty}$, or, dually, the edges of $\Sigma^{*}$ in the boundary of $v_{\infty}$, the number of regions is at least two.

The boundary of each region is a copath in $\Sigma$, and hence the sum of $\theta$ over each boundary is at least $2 \pi$. Sum over all boundaries to obtain

$$
2 \pi r \leq \sum_{e \in E^{\prime \prime}} 2 \theta_{e} .
$$

Indeed, each edge in $E^{\prime \prime}$ appears in at most 2 boundaries. Equality holds if and only if every edge is contained in a boundary and each region contains only one vertex or all vertices but one. This means that equality holds if and only if $E^{\prime \prime}=E$ or $E^{\prime \prime}=\partial^{*} v$ for some vertex $v$; equivalently, if and only if $F^{\prime}=\emptyset$ or $F^{\prime}=F \backslash F_{\infty}$.

Thus, we have shown that

$$
2 \pi\left(\left|E^{\prime \prime}\right|-\left|F^{\prime \prime}\right|\right) \leq \sum_{e \in E^{\prime \prime}} 2 \theta_{e}-2 \pi(n+1),
$$

with equality if and only if $F^{\prime}=\emptyset$ or $F^{\prime}=F \backslash F_{\infty}$.

Equation (25) and Euler's formula,

$$
|F|-|E|+|V|=2,
$$

imply

$$
2 \pi(|F|-|E|)=-\sum_{e \in E} 2 \theta_{e}+4 \pi .
$$

Add equation (27) and inequality (26) to obtain

$$
2 \pi\left(\left|F^{\prime}\right|-\left|E^{\prime}\right|\right) \leq-\sum_{e \in E^{\prime}} 2 \theta_{e}-2 \pi(n-1),
$$

or, because $\theta^{*}=\pi-\theta$,

$$
2 \pi\left|F^{\prime}\right| \leq \sum_{e \in E^{\prime}} 2 \theta_{e}^{*}+2 \pi(1-n)
$$

Equality holds if and only if $F^{\prime}=\emptyset$ or $F^{\prime}=F \backslash F_{\infty}$. Since $n \geq 1$, and $n=1$ if $F^{\prime}=F \backslash F_{\infty}$, we have deduced conditions (ii) and (iii) of Proposition 5 . This completes the proof of Rivin's theorem.

5.3. Higher genus. Proof of Theorem 4. In this section, proceeding in a similar way as in the last section, we derive Theorem 4 from Theorem 3

In one way, the higher genus case is simpler than the spherical case. We do not have to transfer the problem to the plane by a stereographic projection. We may use Theorem 3 directly. For this reason, we do not have to assume the cellular surface to be polytopal.

In another way this case is more complicated. When a graph is embedded in a surface of genus $g \geq 1$, the dimension $c$ of its cycle space and the number $r$ of regions into which the graph separates the surface are not related by the simple formula $c=r-1$. Instead, the following proposition holds. 
Proposition 6. Suppose a nonempty graph $\Gamma=(V, E)$ is embedded in a closed surface of genus $g$ and separates the surface into $r$ regions. Then

$$
r-|E|+|V|=2-2 g+\sum_{j=1}^{r} h_{j},
$$

where $h_{j}$ is the dimension of the first $\mathbb{Z}_{2}$-homology group of the $j^{\text {th }}$ region.

(The graph $\Gamma$ is nonempty if $V \neq \emptyset$.) If all regions are simply connected, then all $h_{j}=0$, and (28) is Euler's equation for a surface of genus $g$. A multiply connected region can be turned into a simply connected one by adding $h_{j}$ edges that do not separate the region. A formal proof of Proposition [6 is given by Giblin Gib77. ch. 9]. It is an application of the long exact sequence of relative homology and Lefschetz duality.

First, we show that the condition of Theorem 4 is necessary for the existence of a circle pattern. For $g=1$, the flat case, this follows from equations (23) and (24) as in the preceding section. (See Figure 7) For $g>1$, one has instead

$$
\sum\left(\pi-\alpha_{j}-\beta_{j}-\gamma_{j}\right)=2 \pi+A
$$

and

$$
\theta_{j}=\pi-A_{j}-\alpha_{j}-\beta_{j},
$$

where $A$ is the area enclosed by $\gamma$ and $A_{j}$ is the area of the triangle with angles $\alpha_{j}$, $\beta_{j}$ and $\theta_{j}$. Hence, $\sum \theta_{j}=2 \pi+\sum \gamma_{j}+A-\sum A_{j}$, and therefore $\sum \theta_{j} \geq 2 \pi$, with equality if and only if $\gamma$ is the coboundary of a single vertex.

In the following, we show that the condition of Theorem 4 is also sufficient for the existence of a circle pattern. Suppose the conditions of Theorem 4 are satisfied. We deduce the conditions of Theorem 3 Thus, let $F^{\prime}$ be a nonempty subset of $F$ and let $E^{\prime}$ be the set of edges incident with any face in $F^{\prime}$. Let $F^{\prime \prime}=F \backslash F^{\prime}$, $E^{\prime \prime}=E \backslash E^{\prime}$, and consider the graph $\Gamma=\left(F^{\prime \prime}, E^{\prime \prime}\right)$ in the dual cellular surface $\Sigma^{*}$. This graph is nonempty if $F^{\prime} \neq F$. In that case, Proposition [ implies

$$
\left|E^{\prime \prime}\right|-\left|F^{\prime \prime}\right|=2 g-2+r-\sum_{j=1}^{r} h_{j} .
$$

Suppose we cut the dual surface $\Sigma^{*}$ along the (dual) edges in $E^{\prime \prime}$. This would separate the surface into pieces. By the boundary of one such piece, we mean the boundary after having cut the surface, not the boundary of the corresponding region in the surface. For example, if an edge $e \in E^{\prime \prime}$ has the same region on either side, the boundary of that piece contains $e$ twice. Thus, every edge of $E^{\prime \prime}$ appears exactly twice in the boundaries of the pieces into which the surface separates. Hence, if $\gamma_{j}$ is the boundary of the $j^{\text {th }}$ region, then

$$
\sum_{j=1}^{r}\left(\sum_{e \in \gamma_{j}} \theta_{e}\right)=\sum_{e \in E^{\prime \prime}} 2 \theta_{e}
$$

By the condition of Theorem 4, for a simply connected piece, i.e., for one with $h_{j}=0$, the sum of $\theta$ over its boundary is at least $2 \pi$, and equal to $2 \pi$ only if the piece consists of a single vertex. Hence, we have

$$
2 \pi\left(r-\sum_{j=1}^{r} h_{j}\right)<\sum_{e \in E^{\prime \prime}} 2 \theta_{e} .
$$


Equality could hold only if each region consisted of a single vertex. But this would imply $E^{\prime \prime}=E$, or $E^{\prime}=\emptyset$, contradicting the assumption that $F^{\prime}$ was nonempty. If $F^{\prime \prime}$ is nonempty, equation (29) implies

$$
2 \pi\left(\left|E^{\prime \prime}\right|-\left|F^{\prime \prime}\right|\right)<2 \pi(2 g-2)+\sum_{e \in E^{\prime \prime}} 2 \theta_{e} .
$$

If $F^{\prime \prime}$ is empty (and hence also $E^{\prime \prime}$ ), this inequality is trivially true if $g>1$, and equality holds if $g=1$.

Because there is no curvature in the vertices, equation (25) holds here as well. With Euler's formula,

$$
|F|-|E|+|V|=2-2 g,
$$

this implies

$$
2 \pi(|F|-|E|)=2 \pi(2-2 g)-\sum_{e \in E} 2 \theta_{e} .
$$

Add this equation to inequality (30) to obtain

$$
2 \pi\left(\left|F^{\prime}\right|-\left|E^{\prime}\right|\right) \leq-\sum_{e \in E^{\prime}} 2 \theta_{e},
$$

with equality if and only if $F^{\prime}=F$ and $g=1$. Since $\theta^{*}=\pi-\theta$, this inequality is equivalent to

$$
2 \pi\left|F^{\prime}\right| \leq \sum_{e \in E^{\prime}} 2 \theta_{e}^{*}
$$

This completes the proof of Theorem 4 .

\section{OTHER VARIATIONAL PRINCIPLES}

In this section, we derive the variational principles of Colin de Verdière, Brägger, and Rivin from ours. For circle packings, Colin de Verdière's functionals are obtained from our functionals $S_{E u c}$ and $S_{\text {hyp }}$ for orthogonally intersecting circles by minimizing with respect to the radii of those circles that do not appear in the packing. The derivation of Brägger's and Rivin's functionals involves a Legendre transformation of $S_{E u c}$. Similarly, a Legendre transformation of $S_{h y p}$ yields a functional which seems to be related to Leibon's.

6.1. Colin de Verdière's functionals. Colin de Verdière considers circle packings in which the circles correspond to the vertices of a triangulation. He considers the 1 -form

$$
\omega=\alpha d u+\beta d v+\gamma d w
$$

on the space of Euclidean triangles, where

$$
u=\log x, \quad v=\log y, \quad w=\log z
$$

and $x, y, z$ and $\alpha, \beta, \gamma$ are as shown in Figure 8

It turns out that $d \omega=0$; hence one may integrate. Define the function $f_{\alpha_{0}, \beta_{0}, \gamma_{0}}$ on $\mathbb{R}^{3}$ by

$$
f_{\alpha_{0}, \beta_{0}, \gamma_{0}}(u, v, w)=\int^{(u, v, w)}\left(\alpha_{0}-\alpha\right) d u+\left(\beta_{0}-\beta\right) d v+\left(\gamma_{0}-\gamma\right) d w
$$

The initial point of the integration does not matter.

Suppose we are given a triangulation and a coherent angle system for it. Here, a coherent angle system is a positive function on the set of angles of the triangles, 


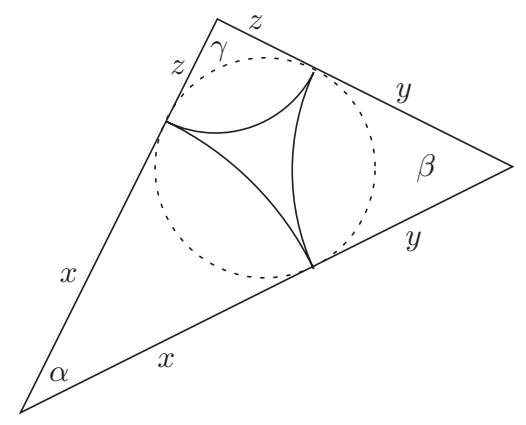

Figure 8. A Euclidean triangle

such that the sum in each triangle is $\pi$, and the sum around each vertex is $2 \pi$. For a function $\rho$ on the vertices of the triangulation, Colin de Verdière's functional for Euclidean circle packings is

$$
S_{C d V}(\rho)=\sum f_{\alpha_{0}, \beta_{0}, \gamma_{0}}(u, v, w),
$$

where the sum is taken over all triangles, $u, v$, and $w$ are the values of $\rho$ on the vertices of each triangle, and $\alpha_{0}, \beta_{0}$, and $\gamma_{0}$ are the corresponding angles of the coherent angle system.

The critical points of this functional correspond to the logarithmic radii of a circle packing.

The hyperbolic case is treated in the same way, except that now

$$
u=\log \tanh (x / 2), \quad v=\log \tanh (y / 2), \quad w=\log \tanh (z / 2),
$$

and in the definition of a coherent angle system, it is required that the sum of the angles in a triangle is less than $\pi$.

To treat circle packings with our functionals, we consider circle patterns with orthogonally intersecting circles on the medial decomposition of the triangulation (see Figure 3).

Suppose $\Sigma_{0}$ is a triangulation. Let $\Sigma$ be the medial decomposition. Set $\theta_{e}=\pi / 2$ for all edges $e$. We will consider only the case of closed surfaces; so let $\Phi_{f}=2 \pi$ for all faces $f$. The faces of $\Sigma$ are of two types: those that correspond to faces of $\Sigma_{0}$ and those that correspond to vertices of $\Sigma_{0}$. Let $F=F_{1} \cup F_{2}$, where $F_{1}$ contains the faces of the first type and $F_{2}$ contains the faces of the second type.

Consider $S_{E u c}$ as function on $\mathbb{R}^{F_{1}} \times \mathbb{R}^{F_{2}}$ and define $S_{2}: \mathbb{R}^{F_{2}} \rightarrow \mathbb{R}$,

$$
S_{2}\left(\rho_{2}\right)=\min _{\rho_{1} \in \mathbb{R}^{F_{1}}} S_{E u c}\left(\rho_{1}, \rho_{2}\right) .
$$

Hence, $S_{2}\left(\rho_{2}\right)=S_{E u c}\left(\rho_{1}, \rho_{2}\right)$, where $\rho_{1} \in \mathbb{R}^{F_{1}}$ is determined as follows. Suppose $f \in F_{1}$ and $f_{a}, f_{b}, f_{c} \in F_{2}$ are the neighboring faces of $f$. Construct the Euclidean triangle whose sides are

$$
e^{\rho_{2} f_{a}}+e^{\rho_{2} f_{b}}, \quad e^{\rho_{2} f_{b}}+e^{\rho_{2} f_{c}}, \quad \text { and } e^{\rho_{2} f_{c}}+e^{\rho_{2} f_{a}} .
$$

Let $\rho_{1}(f)$ be the logarithmic radius of the inscribed circle.

For $\rho_{2} \in \mathbb{R}^{F_{2}}$, and $\rho_{1}$ the corresponding point in $\mathbb{R}^{F_{1}}$,

$$
\frac{\partial S_{E u c}}{\partial \rho_{1}}\left(\rho_{1}, \rho_{2}\right)=0 \text { and } \frac{\partial S_{E u c}}{\partial \rho_{2}}\left(\rho_{1}, \rho_{2}\right)=\frac{\partial S_{2}}{\partial \rho_{2}}\left(\rho_{2}\right) \text {. }
$$


Consider $S_{C d V}$ as a function on $\mathbb{R}^{F_{2}}$. It is not hard to see that $d S_{2}=d S_{C d V}$. This implies that Colin de Verdière's Euclidean functional is, up to an additive constant, equal to $S_{2}$.

Colin de Verdière's functional for hyperbolic circle packings can be derived from $S_{\text {hyp }}$ in the same way.

6.2. A Legendre transformation of the Euclidean functional. Consider a function $S: \mathbb{R}^{F} \rightarrow \mathbb{R}$ of the form

$$
S(\rho)=\sum_{f_{j} \circ \dot{\leftrightarrow}_{\circ} \circ f_{k}} F_{e}\left(\rho_{f_{k}}-\rho_{f_{j}}\right)+\sum_{\circ f_{j}} G_{f}\left(\rho_{f_{j}}\right),
$$

where the first sum is taken over all oriented edges $e$ and the second sum is taken over all faces $f$. The functional $S_{E u c}$ is of this form with

$$
F_{e}(x)=\operatorname{ImLi}_{2}\left(e^{x+i \theta_{e}}\right)
$$

and

$$
G_{f}(x)=\left(\Phi_{f}-\sum \theta_{e}^{*}\right) x
$$

where the sum is taken over all edges in the boundary of $f$.

Define the function $S^{L}: \mathbb{R}^{\vec{E}} \times \mathbb{R}^{F} \rightarrow \mathbb{R}$ by

$$
S^{L}(v, \rho)=\sum_{f_{j} \circ \mathfrak{x}_{\bullet} \circ f_{k}} F_{e}\left(v_{e}\right)+\sum_{\circ f_{j}} G_{f_{j}}\left(\rho_{f_{j}}\right) .
$$

The " $L$ " in the superscript stands for "Lagrange". We do not assume that $v$ is antisymmetric, i.e., that $v_{e}=v_{-e}$. Clearly, the critical points of $S$ correspond to the critical points of $S^{L}$ under the constraints

$$
v_{e}=\rho_{f_{k}}-\rho_{f_{j}},
$$

where $f_{j}$ and $f_{k}$ are the faces to the left and to the right of $e$, respectively.

Definition. Suppose $F$ is a smooth and strictly convex real function on some open interval. Then

$$
y=F^{\prime}(x)
$$

defines a smooth coordinate transformation. The Legendre transform of $F$ is

$$
\tilde{F}(y)=x y-F(x),
$$

where $x$ is related to $y$ by (31).

Note that

$$
\tilde{F}^{\prime}(y)=\frac{d \tilde{F}}{d y}=\frac{d x}{d y} y+x-\frac{d F}{d x} \frac{d x}{d y}=x .
$$

It follows that $F$ is the Legendre transform of $\tilde{F}$.

Now let $\tilde{F}_{e}$ be the Legendre transform of $F_{e}$ and define the function $S^{H}$ by

$$
S^{H}(\varphi, \rho)=\sum_{f_{j} \circ \dot{\boldsymbol{x}}_{\bullet} \circ f_{k}}\left(\varphi_{e}\left(\rho_{f_{k}}-\rho_{f_{j}}\right)-\tilde{F}_{e}\left(\varphi_{e}\right)\right)+\sum_{\circ j} G\left(\rho_{f_{j}}\right) .
$$

The " $H$ " in the superscript stands for "Hamilton". If $\varphi$ and $v$ are related by $\varphi_{e}=$ $F_{e}^{\prime}\left(v_{e}\right)$, then $S^{H}(\varphi, \rho)=S^{L}(v, \rho)$. Remarkably, the critical points of $S$ correspond to critical points of $S^{H}$, where $\rho$ and $\varphi$ may be varied independently: 
Proposition 7. If $(\varphi, \rho)$ is a critical point of $S^{H}$, then $\rho$ is a critical point of $S$.

Conversely, suppose $\rho$ is a critical point of $S$. Let $\varphi_{e}=F_{e}^{\prime}\left(\rho_{f_{k}}-\rho_{f_{j}}\right)$, where $f_{j}$ and $f_{k}$ are the faces to the left and right of $e$. Then $(\varphi, \rho)$ is a critical point of $S^{H}$.

Proof. We have $\partial S^{H} / \partial \varphi_{e}=\rho_{f_{k}}-\rho_{f_{k}}-\tilde{F}_{e}^{\prime}\left(\varphi_{e}\right)$. So all partial derivatives with respect to the variables $\varphi_{e}$ vanish if and only if $\varphi$ and $\rho$ are related by $\rho_{f_{k}}-\rho_{f_{k}}=$ $\tilde{F}_{e}^{\prime}\left(\varphi_{e}\right)$, or, equivalently, $\varphi_{e}=F_{e}^{\prime}\left(\rho_{f_{k}}-\rho_{f_{k}}\right)$. In this case, $S^{H}(\varphi, \rho)=S(\rho)$.

Now consider, in particular, the Euclidean functional $S_{E u c}$. The variables $\rho$ and $\varphi$ are related by equation (7). The inverse relation is

$$
\rho_{f_{k}}-\rho_{f_{j}}=\log \frac{\sin \varphi_{e}}{\sin \left(\varphi_{e}+\theta_{e}\right)} .
$$

For the functional in Hamiltonian form, identity (39) of the appendix implies

$$
\begin{array}{r}
S_{E u c}^{H}(\varphi, \rho)=\sum_{f_{j} \circ \mathfrak{R}_{\circ} \circ f_{k}}\left(\varphi_{e}\left(\rho_{f_{k}}-\rho_{f_{j}}\right)+\frac{1}{2}\left(\mathrm{Cl}_{2}\left(2 \varphi_{e}\right)-\mathrm{Cl}_{2}\left(2 \varphi_{e}+2 \theta_{e}\right)+\mathrm{Cl}_{2}\left(2 \theta_{e}\right)\right)\right) \\
+\sum_{\circ f}\left(\Phi_{f}-\sum_{e \in \partial f} \theta_{e}^{*}\right) \rho_{f} .
\end{array}
$$

Note that $S_{E u c}^{H}$ depends linearly on $\rho$. Collecting the coefficients of each $\rho_{f}$, one finds that if $\varphi$ is a coherent angle system, then $S_{E u c}^{H}(\varphi, \rho)$ does not depend on $\rho$ at all. Therefore, this leads to a new variational principle, where the functional depends only on the angles, and the variation is constrained to the space of coherent angle systems.

Proposition 8. If $\varphi$ is a coherent angle system, then $S_{E u c}^{H}(\varphi, \rho)=\widehat{S}_{E u c}^{H}(\varphi)$, where

$$
\widehat{S}_{E u c}^{H}(\varphi)=\sum\left(\mathrm{Cl}_{2}\left(2 \varphi_{e}\right)+\frac{1}{2} \mathrm{Cl}_{2}\left(2 \theta_{e}\right)\right)
$$

and the sum is taken over all oriented edges e.

If $\varphi$ is a critical point of $\widehat{S}_{E u c}^{H}$ under variations in the space of coherent angle systems, then the equations (32) form a consistent system of linear equations for the $\rho_{f}$. They define a function $\rho \in \mathbb{R}^{F}$ up to an additive constant. This $\rho$ is a critical point of $S_{E u c}$.

Conversely, any critical point of $S_{E u c}$ yields, via equation (7), a coherent angle system which is a critical point of $\widehat{S}_{E u c}^{H}$ under variations in the space of coherent angle systems.

Proof. To derive equation (33), note that, if $\varphi$ is a coherent angle system, then $\varphi_{e}+\theta_{e}=\pi-\varphi_{-e}$. Hence $\mathrm{Cl}_{2}\left(2 \varphi_{e}+2 \theta_{e}\right)=-\mathrm{Cl}_{2}\left(2 \varphi_{-e}\right)$.

Now suppose $\varphi$ is a critical point of $\widehat{S}_{E u c}^{H}$ under variations in the space of coherent angle systems. The partial derivatives of $\widehat{S}_{E u c}^{H}$ are

$$
\frac{\partial \widehat{S}_{E u c}^{H}}{\partial \varphi_{e}}=-2 \log \left(2 \sin \varphi_{e}\right)
$$

The tangent space to the space of coherent angle systems is spanned by vectors of the form

$$
\sum_{e \in \gamma}\left(\frac{\partial}{\partial \varphi_{e}}-\frac{\partial}{\partial \varphi_{-e}}\right)
$$


where $\gamma$ is some cocycle. (If we add to $\varphi_{e}$, we have to subtract the same amount from $\varphi_{-e}$. But the sum of $\varphi$ around each face has to remain constant; so we step from one face to the next, adding and subtracting along some cocycle.)

Since $\varphi$ is a critical point under variations in the space of coherent angle systems,

$$
\sum_{e \in \gamma} \log \frac{\sin \varphi_{e}}{\sin \left(\varphi_{e}+\theta_{e}\right)}=0
$$

for all cocycles $\gamma$. Hence, equations (32) are compatible. It is now easy to complete the proof.

6.3. Brägger's functional and Rivin's functional. Like Colin de Verdière, Brägger Brä92 considers circle packings in which the circles correspond to the vertices of a triangulation. A coherent angle system in the sense of section 6.1 is clearly equivalent to a coherent angle system in the sense of that article. Thus, Brägger's functional is seen to be equal to $\widehat{S}_{E u c}^{H} / 2$, up to an additive constant.

Rivin [Riv94] considers Euclidean circle patterns with arbitrary prescribed intersection angles. The pattern of intersection is determined by a triangulation. Circles correspond to faces of the triangulation. However, since Rivin allows intersection angles $\theta=0$, adjacent triangles may in effect belong to the same circle. Thus, cellular decompositions with non-triangular cases may be treated by first dissecting all faces into triangles. Rivin treats cone-like singularities at the vertices but not at the centers of the circles. His functional is, up to an additive constant, equal to $\widehat{S}_{E u c}^{H} / 2$.

6.4. A Legendre transformation of the hyperbolic functional. Now, consider a function $S: \mathbb{R}^{F} \rightarrow \mathbb{R}$ of the form

$$
S(\rho)=\sum_{f_{j} \circ \mathfrak{\rho}_{\circ f_{k}}}\left(F_{e}^{-}\left(\rho_{f_{k}}-\rho_{f_{j}}\right)+F_{e}^{+}\left(\rho_{f_{j}}+\rho_{f_{k}}\right)\right)+\sum_{\circ f} G_{f}\left(\rho_{f}\right) .
$$

Here, the first sum is taken over all non-oriented edges $e$, and $f_{j}$ and $f_{k}$ are the faces on either side of $e$. We assume that $F^{-}$is an even function, so that it does not matter which face is which. The second sum is taken over all faces $f$. The functional $S_{h y p}$ is of this form with

$$
F_{e}^{-}(x)=F_{e}^{+}(x)=\operatorname{ImLi}_{2}\left(e^{x+i \theta_{e}}\right)+\operatorname{ImLi}_{2}\left(e^{-x+i \theta_{e}}\right)
$$

and

$$
G_{f}(x)=\Phi_{f} x
$$

The space $\mathbb{R}^{\vec{E}}$ of functions on non-oriented edges splits into the space of antisymmetric and symmetric functions,

$$
\mathbb{R}^{\vec{E}}=\operatorname{Alt}(\vec{E}) \oplus \operatorname{Sym}(\vec{E}),
$$

where

and

$$
\operatorname{Alt}(\vec{E})=\left\{v \in \mathbb{R}^{\vec{E}} \mid v_{-e}=-v_{e}\right\}
$$

$$
\operatorname{Sym}(\vec{E})=\left\{w \in \mathbb{R}^{\vec{E}} \mid w_{-e}=w_{e}\right\} .
$$

Define the function $S^{L}: \operatorname{Alt}(\vec{E}) \times \operatorname{Sym}(\vec{E}) \times \mathbb{R}^{F} \rightarrow \mathbb{R}$ by

$$
S^{L}(v, w, \rho)=\sum_{f_{j} \circ \varrho_{\circ} f_{k}}\left(F_{e}^{-}(v)+F_{e}^{+}(w)\right)+\sum_{\circ f} G_{f}\left(\rho_{f}\right) .
$$


Suppose $F^{-}$and $F^{+}$are smooth and strictly convex. The coordinates conjugate to $v_{e}$ and $w_{e}$ are $p_{e}=F^{-\prime}\left(v_{e}\right)$ and $s_{e}=F^{+\prime}\left(w_{e}\right)$. Clearly, $p_{-e}=-p_{e}$ and $s_{-e}=s_{e}$. Let $\tilde{F}^{-}$and $\tilde{F}^{+}$be the Legendre transforms of $F^{-}$and $F^{+}$. For $p$ and $s$ in suitable subsets of $\operatorname{Alt}(\vec{E})$ and $\operatorname{Sym}(\vec{E})$, define

$$
\begin{aligned}
S^{H}(p, s, \rho)= & \sum_{f_{j} \circ \dot{\bullet}_{\circ f_{k}}}\left(p_{e}\left(\rho_{f_{k}}-\rho_{f_{j}}\right)-\tilde{F}_{e}^{-}(p)+s_{e}\left(\rho_{f_{k}}+\rho_{f_{j}}\right)-\tilde{F}_{e}^{+}(s)\right) \\
& +\sum_{\circ f} G_{f}\left(\rho_{f}\right) .
\end{aligned}
$$

If $p$ and $s$ are related to $\rho$ by

$$
p_{e}=F_{e}^{-\prime}\left(\rho_{f_{k}}-\rho_{f_{j}}\right), \quad s_{e}=F_{e}^{+\prime}\left(\rho_{f_{k}}+\rho_{f_{j}}\right),
$$

where $f_{j}$ and $f_{k}$ are the faces to the left and right of $e$, then $S^{H}(p, s, \rho)=S(\rho)$. Again, the critical points of $S$ correspond to critical points of $S^{H}$, where $p, s$, and $\rho$ vary independently. The following proposition is proved in the same way as Proposition 7 .

Proposition 9. If $(p, s, \rho)$ is a critical point of $S^{H}$, subject only to the constraints $p_{-e}=-p_{e}$ and $s_{-e}=s_{e}$, then $\rho$ is a critical point of $S$.

Conversely, suppose $\rho$ is a critical point of $S$. Define $p$ and $s$ by equations (34). Then $(p, s, \rho)$ is a critical point of $S^{H}$.

Now consider the functional $S_{h y p}$. The variables $p$ and $s$ are related to $\rho$ by equations (15) and (16). The Hamiltonian form of the functional follows from equation (40) of the appendix:

$$
\begin{aligned}
S_{h y p}^{H}(p, s, \rho)= & \sum_{f_{j} \circ \dot{\emptyset}_{0}}\left(p_{e}\left(\rho_{f_{k}}-\rho_{f_{j}}\right)+\mathrm{Cl}_{2}\left(\theta_{e}^{*}+p_{e}\right)+\mathrm{Cl}_{2}\left(\theta_{e}^{*}-p_{e}\right)\right. \\
+ & s_{e}\left(\rho_{f_{j}}+\rho_{f_{k}}\right)+\mathrm{Cl}_{2}\left(\theta_{e}^{*}+s_{e}\right)+\mathrm{Cl}_{2}\left(\theta_{e}^{*}-s_{e}\right) \\
& \left.-2 \mathrm{Cl}_{2}\left(2 \theta_{e}^{*}\right)\right)+\sum_{\circ f} \Phi_{f} \rho_{f} .
\end{aligned}
$$

(See equation (40) of the appendix.)

Introduce the variables

on oriented edges instead of $(p, s)$ :

$$
\varphi_{e}=\frac{1}{2}\left(p_{e}-s_{e}\right)
$$

$$
S_{\text {hyp }}^{H}(\varphi, \rho)=S_{\text {hyp }}^{H}(p, s, \rho) .
$$

This functional is linear in $\rho$. As in the Euclidean case, collecting the coefficients of each $\rho_{f}$, one observes that at a critical point $\varphi$ is a coherent angle system. Also, if $\varphi$ is a coherent angle system, then $S_{h y p}^{H}$ does not depend on $\rho$. (Remember that in the hyperbolic case, coherent angle systems satisfy $\varphi_{e}+\varphi_{-e}<\theta^{*}$ instead of $\varphi_{e}+\varphi_{-e}=\theta^{*}$.) Again, this leads to a different variational principle.

Proposition 10. If $\varphi$ is a coherent angle system, then $S_{\text {hyp }}^{H}=\widehat{S}_{\text {hyp }}^{H}$, where

$$
\begin{aligned}
\widehat{S}_{\text {hyp }}^{H}(\varphi)=\sum\left(\mathrm{Cl}_{2}\left(\theta_{e}^{*}+\varphi_{e}-\varphi_{-e}\right)+\mathrm{Cl}_{2}\left(\theta_{e}^{*}-\varphi_{e}+\varphi_{-e}\right)\right. \\
\left.\quad+\mathrm{Cl}_{2}\left(\theta_{e}^{*}+\varphi_{e}+\varphi_{-e}\right)+\mathrm{Cl}_{2}\left(\theta_{e}^{*}-\varphi_{e}-\varphi_{-e}\right)-2 \mathrm{Cl}_{2}\left(2 \theta_{e}^{*}\right)\right) .
\end{aligned}
$$


The sum is taken over non-oriented edges, or more precisely, over one representative for each pair of oppositely oriented edges.

If $\varphi$ is a critical point of $\widehat{S}_{\text {hyp }}^{H}$ under variations in the space of coherent angle systems, then the equations

$$
\rho_{f}=\frac{1}{2} \log \frac{\sin \left(\frac{\theta^{*}-\varphi_{e}-\varphi_{-e}}{2}\right) \sin \left(\frac{\theta^{*}-\varphi_{e}+\varphi_{-e}}{2}\right)}{\sin \left(\frac{\theta^{*}+\varphi_{e}+\varphi_{-e}}{2}\right) \sin \left(\frac{\theta^{*}+\varphi_{e}-\varphi_{-e}}{2}\right)},
$$

where $f$ is the face to the left of e, coherently define $\rho \in \mathbb{R}^{F}$. This $\rho$ is a critical point of $S_{\text {hyp }}$.

Conversely, suppose $\rho$ is a critical point of $S_{\text {hyp }}$. Then equations (8) define a coherent angle system, which is a critical point of $\widehat{S}_{E u c}^{H}$ under variations in the space of coherent angle systems.

The proof is analogous to the proof of Proposition 8. We would like to remark only on two points.

First, equations (36) are equivalent to equations (8). This follows by a straightforward calculation.

Second, to show that equations (36) are consistent if $\varphi$ is a critical point of $\widehat{S}_{\text {hyp }}^{H}$ under variations in the space of coherent angle systems, proceed as follows. Suppose $f$ is the face to the left of $e$, and $e^{\prime}$ is the next edge in the boundary of $f$. It suffices to show that the value for $\rho_{f}$ obtained from the equation involving $\varphi_{ \pm e}$ is the same as the value obtained from the equation involving $\varphi_{ \pm e^{\prime}}$. The tangential space to the space of coherent angle systems is spanned by variations with $\dot{\varphi}_{e}=-1$, $\dot{\varphi}_{e^{\prime}}=1$ for two consecutive oriented edges $e$ and $e^{\prime}$ in the boundary of a face, and $\dot{\varphi}=0$ otherwise. But $\partial \widehat{S}_{\text {hyp }}^{H} / \partial \varphi_{e}$ is twice the right-hand side of equation (36). This implies the consistency of equations (36)

6.5. Leibon's functional. Like Rivin, Leibon [Lei01 considers circle patterns with arbitrary prescribed intersection angles. The circles correspond to the faces of a triangulation. Whereas Rivin treats the Euclidean case, Leibon treats the hyperbolic case. His functional seems to be related to $\widehat{S}_{h y p}^{H}$, but the precise nature of the relationship remains unclear 3

\section{Appendix: The Dilogarithm function and Clausen's integral}

In this appendix, we collect everything about the dilogarithm and Clausen's integral that is relevant for this paper. A more thorough treatment and an extensive bibliography are contained in Lewin's monograph [Lew81].

For $|z| \leq 1$, the dilogarithm function is defined by the power series

$$
\operatorname{Li}_{2}(z)=\frac{z}{1^{2}}+\frac{z^{2}}{2^{2}}+\frac{z^{3}}{3^{2}}+\ldots
$$

For $|z|<1$

$$
-\log (1-z)=\frac{z}{1}+\frac{z^{2}}{2}+\frac{z^{3}}{3}+\ldots
$$

\footnotetext{
${ }^{3}$ Added in proof: There is another functional (a common ancestor) from which both Leibon's functional and the functional $\widehat{S}_{h y p}^{H}$ can be derived.
} 
and hence

$$
\operatorname{Li}_{2}(z)=-\int_{0}^{z} \frac{\log (1-\zeta)}{\zeta} d \zeta .
$$

In the light of this integral representation, one sees that the dilogarithm can be analytically continued to the complex plane cut from 1 to $\infty$ along the positive real axis.

Clausen's integral $\mathrm{Cl}_{2}(x)$ can be defined by the imaginary part of the dilogarithm on the unit circle:

$$
\begin{aligned}
\mathrm{Cl}_{2}(x) & =\operatorname{Im} \operatorname{Li}_{2}\left(e^{i x}\right) \\
& =\frac{1}{2 i}\left(\operatorname{Li}_{2}\left(e^{i x}\right)-\operatorname{Li}_{2}\left(e^{-i x}\right)\right) .
\end{aligned}
$$

Clausen used the integral representation that we will derive below to define this function, hence the name. We consider Clausen's integral as a real-valued function of a real variable. It is $2 \pi$-periodic and odd. The power series representation of the dilogarithm yields the Fourier series representation for Clausen's integral,

$$
\mathrm{Cl}_{2}(x)=\sum_{n=1}^{\infty} \frac{\sin (n x)}{n^{2}} .
$$

Substitute $\zeta=e^{i \xi}$ in the integral representation of the dilogarithm (37) to obtain, for $0 \leq x \leq 2 \pi$,

$$
\mathrm{Cl}_{2}(x)=-\int_{0}^{x} \log \left(2 \sin \frac{\xi}{2}\right) d \xi .
$$

Clausen's integral is almost the same as Milnor's Lobachevski function

$$
\pi(x)=-\int_{0}^{x} \log |2 \sin \xi| d \xi=\frac{1}{2} \mathrm{Cl}_{2}(2 x) .
$$

Another related function appearing in the literature, also called a Lobachevski function, is

$$
\mathrm{L}(x)=-\int_{0}^{x} \log (\cos \xi) d \xi=\frac{1}{2} \mathrm{Cl}_{2}(2 x-\pi)+x \log 2 .
$$

We will now derive a formula expressing the imaginary part of the dilogarithm in terms of Clausen's integral not only on the unit circle, but anywhere in the complex plane. Suppose $x$ is real and $0<\theta<2 \pi$. Substitute $\zeta=e^{\xi+i \theta}$ in (37) to obtain

$$
\begin{aligned}
\operatorname{Im~Li}_{2}\left(e^{x+i \theta}\right) & =\frac{1}{2 i}\left(\operatorname{Li}_{2}\left(e^{x+i \theta}\right)-\operatorname{Li}_{2}\left(e^{x-i \theta}\right)\right) \\
& =\frac{1}{2 i} \int_{-\infty}^{x} \log \left(\frac{1-e^{\xi-i \theta}}{1-e^{\xi+i \theta}}\right) d \xi
\end{aligned}
$$

Now substitute

and note that, inversely,

$$
\eta=\frac{1}{2 i} \log \left(\frac{1-e^{\xi-i \theta}}{1-e^{\xi+i \theta}}\right)
$$

Finally, one obtains

$$
\xi=\log \left(\frac{2 \sin \eta}{2 \sin (\eta+\theta)}\right)
$$

$$
\operatorname{Im} \mathrm{Li}_{2}\left(e^{x+i \theta}\right)=y x+\frac{1}{2} \mathrm{Cl}_{2}(2 y)-\frac{1}{2} \mathrm{Cl}_{2}(2 y+2 \theta)+\frac{1}{2} \mathrm{Cl}_{2}(2 \theta)
$$


where

$$
y=\frac{1}{2 i} \log \left(\frac{1-e^{x-i \theta}}{1-e^{x+i \theta}}\right) .
$$

From this, we derive the formula

$$
\begin{aligned}
& \operatorname{Im} \operatorname{Li}_{2}\left(e^{x+i \theta}\right)+\operatorname{Im~Li}_{2}\left(e^{-x+i \theta}\right) \\
& \quad=p x+\mathrm{Cl}_{2}\left(p+\theta^{*}\right)+\mathrm{Cl}_{2}\left(-p+\theta^{*}\right)-\mathrm{Cl}_{2}\left(2 \theta^{*}\right),
\end{aligned}
$$

where $\theta^{*}=\pi-\theta$, and

$$
p=\frac{1}{2 i} \log \frac{\left(1+e^{x+i \theta^{*}}\right)\left(1+e^{-x-i \theta^{*}}\right)}{\left(1+e^{x-i \theta^{*}}\right)\left(1+e^{-x+i \theta^{*}}\right)} .
$$

Finally, note that $p$ and $x$ are related by

$$
\tan \left(\frac{p}{2}\right)=\tanh \left(\frac{x}{2}\right) \tan \left(\frac{\theta^{*}}{2}\right) .
$$

\section{REFERENCES}

[Bow91] B. H. Bowditch, Singular Euclidean structures on surfaces, J. London Math. Soc. (2) 44 (1991), no. 3, 553-565. MR 93i:57014

[Brä92] W. Brägger, Kreispackungen und Triangulierungen, Enseign. Math. 38 (1992), 201217. MR 94b:52032

[BS93] G. R. Brightwell and E. R. Scheinerman, Representations of planar graphs, SIAM J. Discrete Math. 6 (1993), no. 2, 214-229. MR 95d:05043

[BS02] A. I. Bobenko and Yu. B. Suris, Integrable systems on quad-graphs, Internat. Math. Res. Notices 2002, no. 11, 573-612. MR 2003d:37127

[CdV91] Y. Colin de Verdière, Un principe variationnel pour les empilements de cercles, Invent. Math. 104 (1991), 655-669. MR 92h:57020

[DS95] T. Dubejko and K. Stephenson, Circle packing: experiments in discrete analytic function theory, Experiment. Math. 4 (1995), no. 4, 307-348. MR 97f:57027

[FF62] L. R. Ford, Jr. and D. R. Fulkerson, Flows in networks, Princeton University Press, Princeton, NJ, 1962. MR 28:2917

[Gar92] B. T. Garrett, Circle packings and polyhedral surfaces, Discrete Comput. Geom. 8 (1992), 429-440. MR 93g:52014

[Gib77] P. J. Giblin, Graphs, surfaces and homology, Chapman and Hall, London, 1977. MR 55:11235

[HBS+ 99] M. K. Hurdal, P. L. Bowers, K. Stephenson, De Witt L. Sumners, K. Rehm, K. Schaper, and D. A. Rottenberg, Quasi-conformally flat mapping the human cerebellum, Medical Image Computing and Computer-Assisted Intervention-MICCAI '99 (Berlin) (Ch. Taylor and A. Colchester, eds.), Lecture Notes in Computer Science, vol. 1679, Springer-Verlag, 1999, pp. 279-286.

[Koe36] P. Koebe, Kontaktprobleme der konformen Abbildung, Abh. Sächs. Akad. Wiss. Leipzig Math.-Natur. Kl. 88 (1936), 141-164.

[Lei01] G. Leibon, Characterizing the Delaunay decompositions of compact hyperbolic surfaces, Geom. Topol. 6 (2002), 363-391. MR 2003c:52034

[Lew81] L. Lewin, Polylogarithms and associated functions, North Holland, New York, 1981. MR 83b:33019

[Mar01] D. Martindale, Road map for the mind, Scientific American 285 (2001), 13.

[Mer01] Ch. Mercat, Discrete Riemann surfaces and the Ising model, Commun. Math. Phys. 218 (2001), 177-216. MR 2002c:82019

[Moh93] B. Mohar, A polynomial time circle packing algorithm, Discrete Math. 117 (1993), 257-263. MR 94h:52038

[Riv94] I. Rivin, Euclidean structures on simplicial surfaces and hyperbolic volume, Ann. of Math. 139 (1994), 553-580. MR 96h:57010

[Riv96] A characterization of ideal polyhedra in hyperbolic 3-space, Ann. of Math. 143 (1996), 51-70. MR 96i:52008 
VARIATIONAL PRINCIPLES FOR CIRCLE PATTERNS; KOEBE'S THEOREM

[Riv99] Combinatorial optimization in geometry, Preprint arXiv:math.GT/9907032, July 1999, To appear in Adv. in Appl. Math.

[Sac94] H. Sachs, Coin graphs, polyhedra, and conformal mappings, Discrete Math. 134 (1994), 133-138. MR 95j:52020

[Sch92] O. Schramm, How to cage an egg, Invent. Math. 107 (1992), no. 3, 543-560. MR 93c:52009

[Sch02] J. M. Schlenker, Hyperbolic manifolds with polyhedral boundary, Preprint. ArXiv:math.GT/0111136, v.5, September 2002.

[SR34] E. Steinitz and H. Rademacher, Vorlesungen über die Theorie der Polyeder, SpringerVerlag, Berlin, 1934. MR 55:3962 (reprint)

[Ste22] E. Steinitz, Polyeder und Raumeinteilungen, Encyclopädie der mathematischen Wissenschaften, vol. 3 (Geometrie), 1922, Part 3AB12, pp. 1-139.

[Thu] W. P. Thurston, The geometry and topology of three-manifolds, electronic version 1.0 of 1997. A version is currently available from the Mathematical Sciences Research Institute at the URL http://www.msri.org/publications/books/gt3m/.

[Zie95] G. M. Ziegler, Lectures on polytopes, Springer-Verlag, 1995. MR 96a:52011

Institut für Mathematik, MA 8-3, Technische Universität Berlin, Strasse des 17. Juni 136, 10623 Berlin, Germany

E-mail address: bobenko@math.tu-berlin.de

Institut für Mathematik, MA 8-5, Technische Universität Berlin, Strasse des 17. Juni 136, 10623 Berlin, Germany

E-mail address: springb@math.tu-berlin.de 